\title{
A systematic review of post-release programs for women exiting prison with substance-use disorders: assessing current programs and weighing the evidence
}

\author{
Layla Edwards' ${ }^{1}$, Sacha Kendall Jamieson ${ }^{2,5}$, Julia Bowman ${ }^{3,4,5}$, Sungwon Chang ${ }^{6}$, Josie Newton ${ }^{7}$ and
} Elizabeth Sullivan ${ }^{4,8,9^{*}}$ (D)

\begin{abstract}
Background: The rising rates of women in prison is a serious public health issue. Unlike men, women in prison are characterised by significant histories of trauma, poor mental health, and high rates of substance use disorders (SUDs). Recidivism rates of women have also increased exponentially in the last decade, with substance related offences being the most imprisoned offence worldwide. There is a lack of evidence of the effectiveness of postrelease programs for women. The aim of this systematic review is to synthesise and evaluate the evidence on postrelease programs for women exiting prison with SUDs.

Methods: We searched eight scientific databases for empirical original research published in English with no date limitation. Studies with an objective to reduce recidivism for adult women ( $\geqslant 18$ years) with a SUD were included. Study quality was assessed using the revised Cochrane Risk of Bias tool for randomized trials (RoB2) and the Risk of Bias in Non-randomized Studies - of Interventions (ROBINS-I) tools.

Results: Of the 1493 articles, twelve ( $n=3799$ women) met the inclusion criteria. Recidivism was significantly reduced in five (42\%) programs and substance-use was significantly reduced in one (8.3\%) program. Common attributes among programs that reduced recidivism were: transitional, gender-responsive programs; provision of individualised support; providing substance-related therapy, mental health and trauma treatment services. Methodological and reporting biases were common, which impacted our ability to synthesize results further. Recidivism was inconsistently measured across studies further impacting the ability to compare results across studies.
\end{abstract}

\footnotetext{
* Correspondence: E.Sullivan@newcastle.edu.au

${ }^{4}$ Faculty of Health, University of Technology Sydney, Ultimo, NSW 2007, Australia

${ }^{8}$ Acting Deputy Vice Chancellor Research, University of Newcastle, Callaghan, NSW 2308, Australia

Full list of author information is available at the end of the article
}

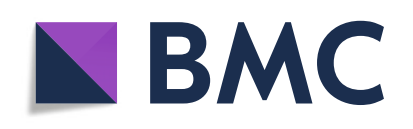

(- The Author(s). 2021 Open Access This article is licensed under a Creative Commons Attribution 4.0 International License, which permits use, sharing, adaptation, distribution and reproduction in any medium or format, as long as you give appropriate credit to the original author(s) and the source, provide a link to the Creative Commons licence, and indicate if changes were made. The images or other third party material in this article are included in the article's Creative Commons licence, unless indicated otherwise in a credit line to the material. If material is not included in the article's Creative Commons licence and your intended use is not permitted by statutory regulation or exceeds the permitted use, you will need to obtain permission directly from the copyright holder. To view a copy of this licence, visit http://creativecommons.org/licenses/by/4.0/. The Creative Commons Public Domain Dedication waiver (http://creativecommons.org/publicdomain/zero/1.0/) applies to the data made available in this article, unless otherwise stated in a credit line to the data. 
Conclusions: Recidivism is a problematic measure of program efficacy because it is inconsistently measured and deficit-focused, unrecognising of women's gains in the post-release period despite lack of tailored programs and significant health and social disadvantages. The current evidence suggests that women benefit from continuity of care from prison to the community, which incorporated gender-responsive programming and individualised case management that targeted co-morbid mental health and SUDs. Future program design should incorporate these attributes of successful programs identified in this review to better address the unique challenges that women with SUDs face when they transition back into the community.

Keywords: Women, Prisoners, Post-release, Transitional, Intervention, Re-entry program, Program evaluation, Substance-related disorders, Recidivism, Systematic review

\section{Introduction}

Incarcerated women are one of the most vulnerable groups in society who, upon entry into prison exhibit a range of complex and inter-related health and social issues (Dumont, Brockmann, Dickman, Alexander, \& Rich, 2012; J. E. Johnson \& Zlotnick, 2008; Kinner \& Young, 2018; Pelissier, Motivans, \& Rounds-Bryant, 2005). Although the proportion of incarcerated women globally is much lower than the proportion of incarcerated men (6.9\% compared to $93.1 \%$, respectively) (Walmsley, 2017), the number of women imprisoned since 2000 continues to increase globally at a rate that is double the rate for the imprisonment of men (Australian Bureau of Statistics, 2018b; J. E. Johnson \& Zlotnick, 2008; B. E. Salem et al., 2013; Walmsley, 2017). There are considerable variations between countries, for example the latest Australian figures show that around 8\% $(n=3587)$ of the prison population is women (Australian Bureau of Statistics, 2018a) and in the United Kingdom this figure was 5\% $(n=7745)$ (Women in Prison, 2017). The United States has the highest total number of women in prison $(n=211,870$, representing $8.7 \%)$ in any one country, as well as the highest prison population rate for women (about 65.7 per 100,000 of the national population) (World Prison Brief, 2018). Comparatively, African countries have a much lower total prison population proportion at $3.4 \%$ (or 3.2 per 100,000 of the national population) (Walmsley, 2017).

\section{Characteristics of women in prison}

Much of this rise is associated with increases in the arrest, prosecution, and incarceration for substance-related offenses (alcohol and other drugs) (Ray, Grommon, Buchanan, Brown, \& Watson, 2017). Unlike men, women are typically imprisoned for non-violent offences; with substance-related offences being the most imprisoned offence worldwide (Australian Bureau of Statistics, 2017a; Begun, Rose, \& LeBel, 2011; Rushforth \& Willis, 2003; World Health Organisation, 2009). The correlation between substance-use and criminal offending has been well researched (Begun et al., 2011; Fearn et al., 2016; H. Johnson, 2006; Moore, Hacker, Oberleitner, \& McKee,
2020) and the evidence shows women to have disproportionately higher rates of substance-use disorders (SUDs) compared to men in prison and compared to women in the general community (Begun et al., 2011). A systematic review across ten countries found upon reception to prison the estimated pooled prevalence of alcohol use disorders for women in prison was $20 \%(95 \% \mathrm{CI}=16$ $24)$ compared to $26 \%(95 \% \mathrm{CI}=23-30)$ for men. The estimated pooled prevalence of drug use disorders was $51 \%(95 \% \mathrm{CI}=43-58)$ for women compared to $30 \%$ (95\% CI $=22-38$ ) for men (Fazel, Yoon, \& Hayes, 2017). Another study reviewed trends in substance-use by gender among people in jail over an 18 year period (19982016) (Bello, Hearing, Salas, Weinstock, \& Linhorst, 2020). Significant differences in substance-use trends was noted: Heroin (36.4\% women vs. $22.0 \%$ men $p<$ $0.0001)$ and stimulants (38.0\% women vs. $19.6 \%$ men, $\mathrm{p}<0.0001$ ) were more strongly preferred by women than men while alcohol $(49.0 \%$ men vs. $29.1 \%$ women, $\mathrm{p}<$ $0.0001)$ and marijuana $(48.7 \%$ men vs. $33.6 \%$ women, $p<0.0001)$ were more strongly preferred by men. There was a low overall prevalence for preference of prescription drugs (8.0\%), however twice as many women strongly preferred this category compared to men $(12.9 \%$ women vs. $6.2 \%$ men, $\mathrm{p}<0.0001$ ) (Bello et al., 2020). Other research has shown that women typically begin SUD treatment with more complex and significant physical, emotional and behavioural needs compared to men (Back et al., 2011; NIDA., 2021). Despite this, women are more likely than men to face multiple barriers affecting access and entry to SUD treatment (Tuchman, 2010).

Along with SUDs, women in prison are characterised by extensive histories of trauma and poor mental health (MH) (Covington, 2001; J. E. Johnson \& Zlotnick, 2008; B. E. Salem et al., 2013; Schonbrun, Johnson, Anderson, Caviness, \& Stein, 2017; Wetton \& Sprackett, 2007; World Health Organisation, 2009). The prevalence of emotional, physical, and sexual abuse is reported between $77 \%$ and $90 \%$ of women in prison respectively (Australian Institute of Family Studies, 2012; Messina \& Grella, 2006). A recent review summarised the literature 
on sexual abuse and mental illness prevalence among samples of incarcerated women (Karlsson \& Zielinski, 2018). Best estimates for sexual abuse were: $50-66 \%$ for child sexual abuse, $28-68 \%$ for adult sexual abuse, and $56-82 \%$ for a lifetime of sexual assault (Karlsson \& Zielinski, 2018). The review highlighted that incarcerated women have significantly greater exposure to sexual victimization compared to national standards, incarcerated men and women in community (Karlsson \& Zielinski, 2018).

Experiences of trauma predispose women for adverse $\mathrm{MH}$ conditions such as post-traumatic stress disorder, depression, anxiety and suicide (Karlsson \& Zielinski, 2018; World Health Organisation, 2009). Women who experienced trauma as a child have a $40 \%$ increase in odds of developing a $\mathrm{MH}$ condition in adulthood (Messina \& Grella, 2006). A meta-analysis of the effect of adverse childhood experiences on health describes the findings of 37 studies and presents the pooled risk of various health conditions (Hughes et al., 2017). The risk of adverse $\mathrm{MH}$ conditions, such as anxiety, depression, and schizophrenia, was found to be about four times higher, as compared to people who experienced less than four adverse childhood experiences (anxiety OR 3.70; depression OR 4.40, schizophrenia OR 3.60). In addition, people with four or more adverse childhood experiences were at higher risk of SUDs with problematic alcohol use nearly six times higher (OR 5.84) and problematic drug use over ten times as high (OR 10.22) (Hughes et al., 2017). Substance dependency among women in prison is significantly higher among women who have experienced childhood abuse and $\mathrm{MH}$ problems $(\mathrm{H}$. Johnson, 2006).

Women are more likely than men to start using substances as a means to alleviate the pain of trauma and to manage existing $\mathrm{MH}$ conditions (Langan \& Pelissier, 2001; Stalans, 2009). Trauma, $\mathrm{MH}$ and substance-use are therefore inter-related factors that can result in cumulative and compounding $\mathrm{MH}$ issues, addiction, and contact with the criminal justice system (see Fig. 1) (Alleyne, 2008; Australian Bureau of Statistics, 2017b; Covington, 2001; Karlsson \& Zielinski, 2018; B. E. Salem et al., 2013).

\section{Exiting prison}

Compared to men, women generally serve short sentences which is a reflection of the minor, non-violent crimes they have been sentenced for (Baldry, 2010; Balyakina et al., 2014; van den Bergh, Gatherer, \& Møller, 2009). When women are released into the community they face many disadvantages including poor continuity of care, inadequate social support, parenting stress, homelessness and poverty, and reduced employment opportunities (Baldry, 2010; Begun, Early, \& Hodge, 2016; B. E. Salem et al., 2013). A notable difference between men and women in prison is that half of

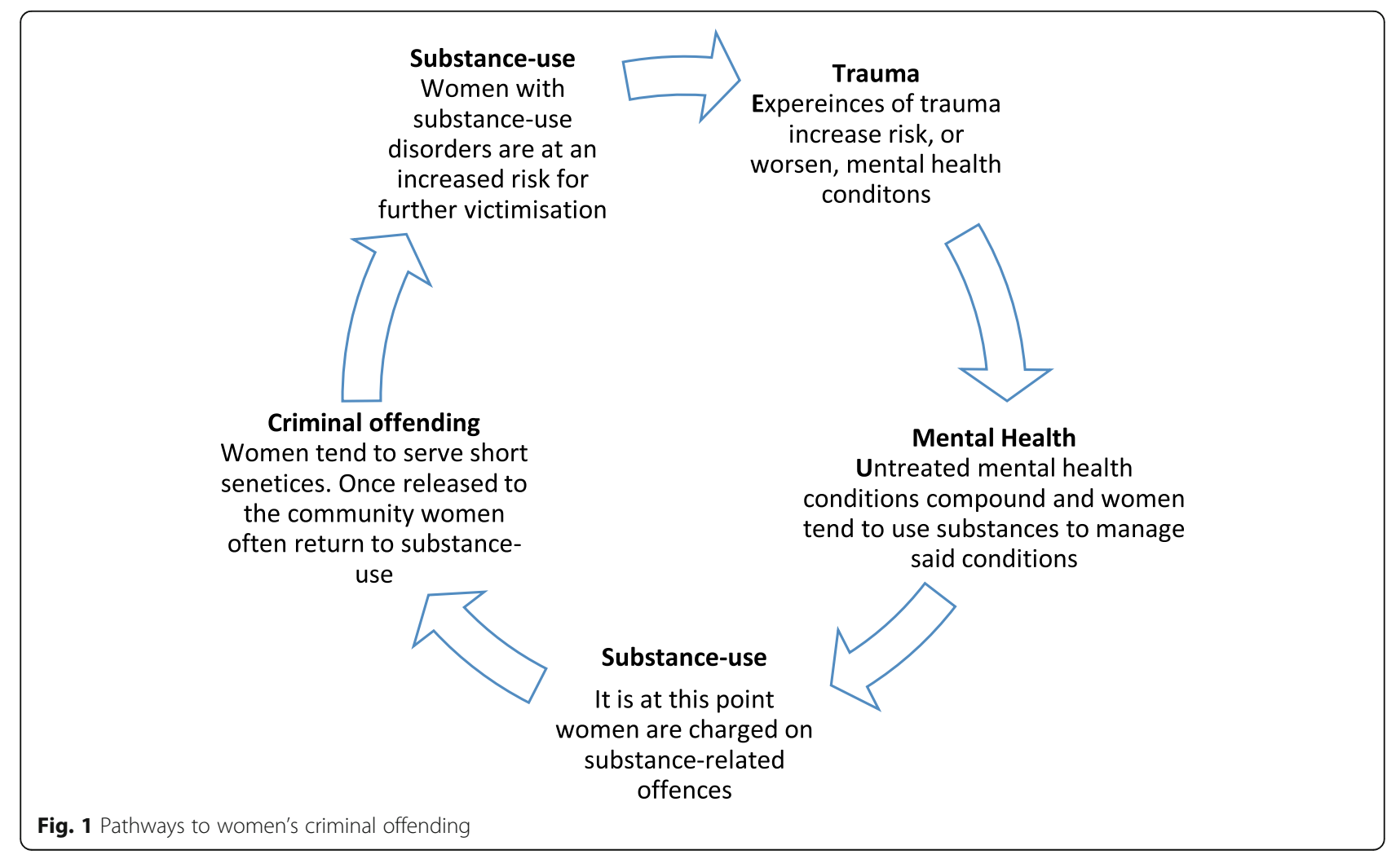


incarcerated women $(54 \%)$ are mothers to dependent children $($ age $<16)$ and were the primary carer of one or more children before incarceration (compared to only $36 \%$ for men) (Australian Institute of Health and Welfare, 2019; Kilroy, 2016). Maternal stress, coupled with the many disadvantages cited, are often barriers to accessing immediate and affordable healthcare and drug and alcohol treatment services. As a result, women with SUDs who are recently released from prison are at a high risk of experiencing an adverse $\mathrm{MH}$ episode, illness and death compared to the general population. The risk of death is especially high in the first month after release, and the causes of death are usually preventable, including suicide, injury, and overdose (Sullivan et al., 2019).

Post-release (also known as re-entry, reintegration, and resettlement) programs are interventions that are delivered in the community. Transitional programs are interventions that start pre-release (in custody) and support people during the transition from prison to community (Baldry, 2010; Borzycki, 2005). Post-release and transitional programs are often evaluated based on a measurement of recidivism. Recidivism is used to measure the proportion of people who go on to reoffend during a pre-defined post-release period (Bartels \& Gaffney, 2011; Sullivan et al., 2019; Urban Institute, n.d.; Yukhnenko, Sridhar, \& Fazel, 2019). A systematic review of recidivism rates, two years post-release for both men and women across 11 countries found re-arrest rates were between $26 \%$ and $60 \%$ and reconviction rates ranged from 20\% to 63\% (Yukhnenko et al., 2019). These recidivism rates suggest that many people with a history of incarceration either do not access, or do not benefit from services and programs during their time in prison, or do not have adequate support or change in social circumstances in the community to prevent reoffendingarrest (Baldry, McDonnell, Maplestone, \& Peeters, 2006).

Despite the growth of the women's prison population, and their profoundly different criminogenic profile compared to incarcerated men, the majority of prison programs available have been designed for men and extended to women with little alteration (Armstrong, Chartrand, \& Baldry, 2005; Bartels \& Gaffney, 2011; Langan \& Pelissier, 2001; Lawlor, Nicholls, \& Sanfilippo, 2008; Suter, Byrne, Byrne, Howells, \& Day, 2002). Emerging evidence indicates that community based programs that are gender-responsive and address criminogenic needs can improve the transition process and minimise recidivism rates post-release (Begun et al., 2016; Borzycki, 2005; Borzycki \& Baldry, 2003; Carlton \& Segrave, 2016). Gender-responsiveness (or genderinformed) refers to programming that explicitly considers the needs that are particularly salient to women. Gender-responsive approaches are trauma-informed and consider the gendered context (or "pathways") of criminal offending (Covington \& Bloom, 2006; Gobeil, Blanchette, \& Stewart, 2016). A meta-analytic review of correctional interventions for women in prison examined whether programs, either gender-informed or gender-neutral, were effective in reducing recidivism (Gobeil et al., 2016). The results demonstrated that participation was associated with $22 \%$ to $35 \%$ greater odds of community success and gender-responsive interventions were significantly more likely to be associated with reductions in recidivism (Gobeil et al., 2016).

Given the proportion of women in prison with SUDs and correlation to reoffending and risk of death postrelease, more research is needed to understand the effectiveness of programs for this population. To-date, there has been no systematic review of the evidence about what is available and "what works" in regard to post-release programs for women with SUDs. The aim of this research is to critically review the available evidence of the effectiveness of community based (post-release and transitional) programs offered to women with SUDs to inform program development to decrease reoffending. Further, as the link between criminal offending and substance-use is well established, we also aim to review the effectiveness of interventions to reduce substance-use outcomes post-release and whether this impacts recidivism. This review addresses the following research questions $(\mathrm{RQ})$ :

RQ1: Are post-release and/or transitional programs effective in reducing recidivism and/or substance-use for women with SUDs post-release?

RQ2: Do those that report a reduction in substance-use also report a reduction in recidivism?

RQ3: What program characteristics are common among programs which report improved recidivism and substance-use outcomes post-release?

\section{Methods}

This systematic review followed the Preferred Reporting Items for Systematic Reviews and Meta-Analyses (PRISMA) guidelines (Moher, Liberati, Tetzlaff, Altman, $\&$ the PRISMA Group, 2009). The systematic review was registered in the PROSPERO database CRD42020162036. The databases PubMed and CINAHL (including MESH terms), Cochrane, EMBASE (including EMTREE terms), Scopus, PsycInfo, ProQuest and SOCIndex were originally searched in September 2019, with no date limitation. The search strategy was split into six core concepts using a combination of words related to "Post-release", "Prison", "Women", and "Interventions". The electronic database searches were supplemented with manual searches of the reference lists from relative articles. Due to the limited number of 
publications found an updated search was conducted in February 2020 following the method by Bramer and Bain (2017), adding search terms related to "Substance use" and "Recidivism" (see Additional File 1).

\section{Eligibility criteria}

Studies included were primary reports of effectiveness trials (i.e., studies of an intervention with a comparator) with an objective to reduce recidivism for adult women $(\geqslant 18$ years) with a known SUD. The program had to be either a post-release or transitional intervention, published in English in a peer-reviewed journal. In this review substance-use included individuals using occasional drugs or alcohol, those who were dependent, or those who had other drug and alcohol related problems prior to their current offence. Studies that included both men and women were included if the results relating to women could be isolated. Due to the limited published studies of women in prison (Baldry, 2010; Borzycki \& Baldry, 2003; Segrave \& Carlton, 2011) there were no limitations by study design or intervention type to ensure identification of all successful programs. Interventions that were pre-release only (only delivered whilst incarcerated), did not focus on women, were mix gendered and did not report gendered data separately, were excluded. Systematic reviews, meta-analyses, protocol papers and studies that did not evaluate a program were also excluded.

\section{Data extraction and quality assessment}

Search results were imported into Endnote X7 software, duplicates removed, and results exported into Covidence online software. Two investigators independently applied eligibility criteria to titles and abstracts and discrepancies identified through the platform were discussed in a face-to-face meeting. Studies that were included were progressed to full-text review where the investigators systematically went through individual articles thoroughly to check eligibility and documented reasons for exclusion. Discrepancies were resolved through face-toface discussion and a third reviewer was approached when needed. The lead reviewer extracted data according to the template for intervention description and replication (TIDieR) checklist and guide (Hoffmann, Glasziou, \& Boutron, 2014) into a Microsoft Excel spreadsheet.

Finally, the investigators independently evaluated the risk of bias of studies using the revised Cochrane Risk of Bias tool for randomized trials (RoB2) (Sterne JAC et al., 2019) and the Risk of Bias in Non-randomized Studies of Interventions (ROBINS-I) tool (Sterne JAC et al., 2016) (See supplementary files 1-2 for full version assessment tools). Each study was scored one point for each criterion that was fully met, half a point $(0.5)$ if a criterion was 'somewhat' met, and zero for criteria that were either not met ('no') or not applicable. Each paper's score was estimated by summing the criteria scores and dividing the total by the number of applicable fields (excluding those criteria which did not apply) and multiplying by 100 . Scores $<0.50$ were characterised as 'low/ moderate quality' and $>0.50$ as 'fair quality'. After the investigators individually assessed studies, they resolved discrepancies through discussion. It should be noted that due to the type of intervention being assessed it was not possible to blind participants, staff, or outcome assessors to participant allocation. We therefore did not score this against the studies performance or detection bias (RoB2 criteria numbers 2.1, 2.2, 4.3; ROBINS-I criteria numbers 6.2).

\section{Data synthesis}

Tables and text were generated to report study and program characteristics and outcomes. An intervention matrix was created, and descriptive numerical analyses were performed using Microsoft Excel.

\section{Results}

The original database search included 1047 citations and the updated search included in 446 citations, resulting in 1493 citations. After the removal of 785 duplicates and 589 articles through title and abstract screening, we reviewed 119 full text articles, of which 105 were excluded as they did not fit the selection criteria. Eleven articles met the criteria with one additional article included following hand-searching, resulting in a total of 12 articles for review (see Fig. 2).

The 12 studies were conducted between 2005 and 2018 with 11 studies from the United States (Chan et al., 2005; Covington, Burke, Keaton, \& Norcott, 2008; Grella \& Rodriguez, 2011; Guydish et al., 2011; J. E. Johnson, Friedmann, Green, Harrington, \& Taxman, 2011; Messina, Burdon, \& Prendergast, 2006; Miller, Miller, \& Barnes, 2016; Needels, James-Burdumy, \& Burghardt, 2005; Nyamathi et al., 2018; Schram \& Morash, 2002; Scott, Dennis, \& Lurigio, 2017) and one from Canada (Farrell-Macdonald, Macswain, Cheverie, Tiesmaki, \& Fischer, 2014) (see Table 1 and Additional file 2). Most studies were either RCTs $(n=5)$ (Guydish et al., 2011; J. E. Johnson et al., 2011; Needels et al., 2005; Nyamathi et al., 2018; Scott et al., 2017) or quasi-experimental studies $(n=4)$ (Chan et al., 2005; Messina et al., 2006; Miller et al., 2016; Schram \& Morash, 2002). There was a total of 4865 participants in the 12 studies with women making up $78 \%$ of participants and ranging in mean age from 30.1 to 39.1 years (excluding one study who did not report mean age (Schram \& Morash, 2002)).

The post-release setting of programs was predominantly community-based (outpatient care) $(n=10)$ (Chan 


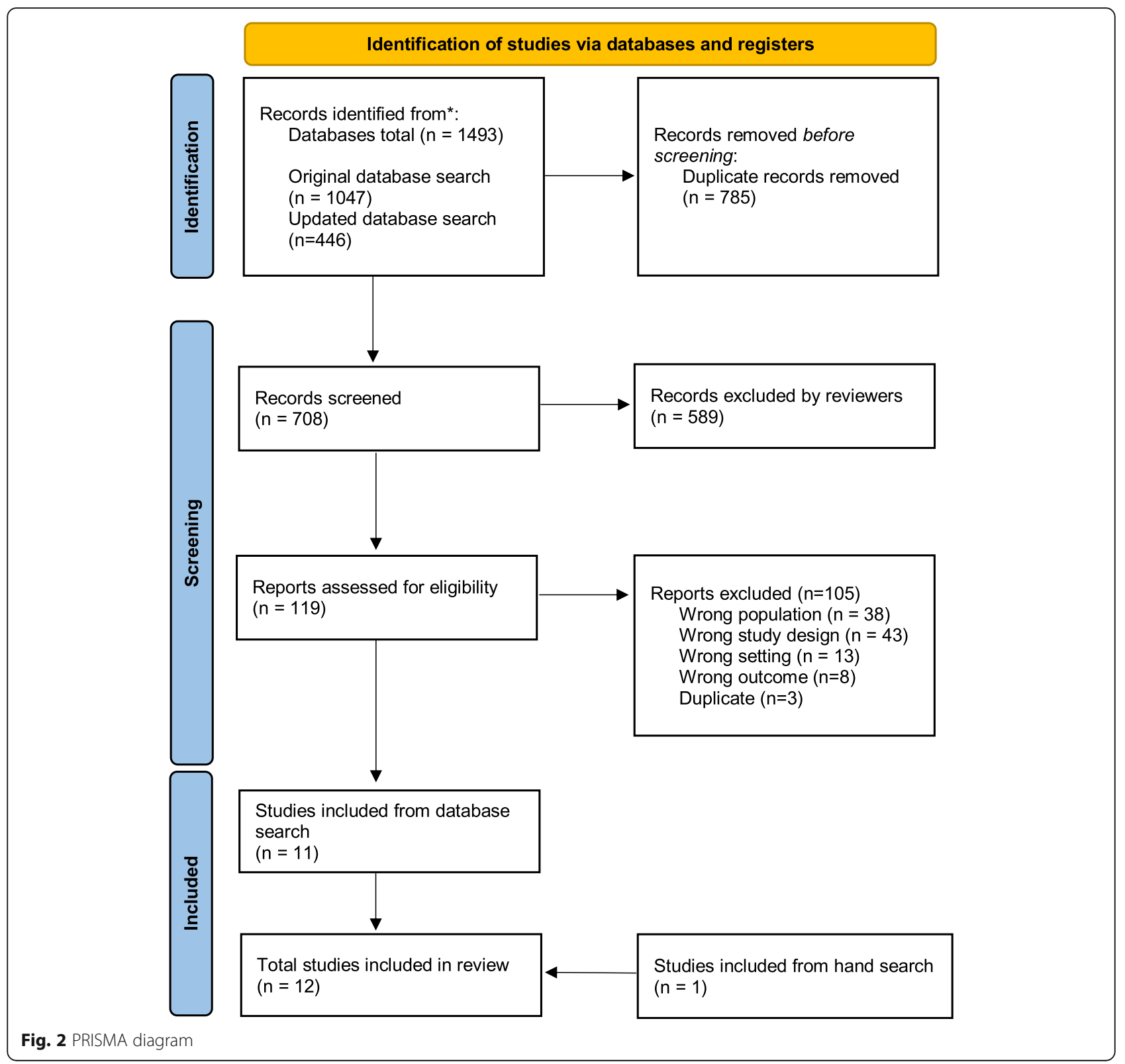

et al., 2005; Farrell-Macdonald et al., 2014; Grella \& Rodriguez, 2011; Guydish et al., 2011; J. E. Johnson et al., 2011; Miller et al., 2016; Needels et al., 2005; Nyamathi et al., 2018; Schram \& Morash, 2002; Scott et al., 2017), with one study occurring in a residential treatment facility (inpatient) (Covington et al., 2008). Most studies included women only $(n=9)$ (Chan et al., 2005; Farrell-Macdonald et al., 2014; Grella \& Rodriguez, 2011; Messina et al., 2006; Nyamathi et al., 2018; Schram \& Morash, 2002), while one accepted women with their children (Covington et al., 2008) and two were mixedgendered (J. E. Johnson et al., 2011; Needels et al., 2005). Seven studies reported parenting characteristics (Chan et al., 2005; Covington et al., 2008; Grella \& Rodriguez,
2011; Guydish et al., 2011; J. E. Johnson et al., 2011; Schram \& Morash, 2002; Scott et al., 2017), of which the proportion of mothers ranged from 63 to $82 \%$ (excluding two studies who reported the average (Messina et al., 2006) and the median (Chan et al., 2005) number of children in their population).

Recidivism was a primary outcome in half (50\%) of the studies (Farrell-Macdonald et al., 2014; Messina et al., 2006; Miller et al., 2016; Needels et al., 2005; Nyamathi et al., 2018; Schram \& Morash, 2002) and a secondary outcome in the remaining studies (50\%) (Chan et al., 2005; Covington et al., 2008; Grella \& Rodriguez, 2011; Guydish et al., 2011; J. E. Johnson et al., 2011; Scott et al., 2017). Other outcomes included: substance-use 
Table 1 Included study characteristics

\begin{tabular}{|c|c|c|}
\hline Characteristics & $\mathrm{N}(\%)$ & Reference \\
\hline Inclusion period, years & $2002-2018$ & $\begin{array}{l}\text { (Chan et al., 2005; Covington et al., 2008; Farrell-Macdonald et al., 2014; Grella \& Rodriguez, 2011; } \\
\text { Guydish et al., 2011; J. E. Johnson et al., 2011; Messina et al., 2006; Miller et al., 2016; Needels et al., 2005; } \\
\text { Nyamathi et al., 2018; Schram \& Morash, 2002; Scott et al., 2017) }\end{array}$ \\
\hline \multicolumn{3}{|l|}{ Country } \\
\hline United States & $11(92 \%)$ & $\begin{array}{l}\text { (Chan et al., 2005; Covington et al., 2008; Grella \& Rodriguez, 2011; Guydish et al., 2011; J. E. Johnson et al., 2011; } \\
\text { Messina et al., 2006; Miller et al., 2016; Needels et al., 2005; Nyamathi et al., 2018; Schram \& Morash, 2002; } \\
\text { Scott et al., 2017) }\end{array}$ \\
\hline Canada & $1(8 \%)$ & (Farrell-Macdonald et al., 2014) \\
\hline \multicolumn{3}{|l|}{ Design } \\
\hline Randomised control trial & $5(42 \%)$ & (Guydish et al., 2011; J. E. Johnson et al., 2011; Needels et al., 2005; Nyamathi et al., 2018; Scott et al., 2017) \\
\hline Quasi-experimental & $4(33 \%)$ & (Chan et al., 2005; Messina et al., 2006; Miller et al., 2016; Schram \& Morash, 2002) \\
\hline $\begin{array}{l}\text { One-group pre/post-test } \\
\text { design }\end{array}$ & $1(8 \%)$ & (Covington et al., 2008) \\
\hline Cohort & $1(8 \%)$ & (Grella \& Rodriguez, 2011) \\
\hline Retrospective & $1(8 \%)$ & (Farrell-Macdonald et al., 2014) \\
\hline \multicolumn{3}{|l|}{ Study population } \\
\hline Female only & $10(83 \%)$ & $\begin{array}{l}\text { (Chan et al., 2005; Covington et al., 2008; Farrell-Macdonald et al., 2014; Grella \& Rodriguez, 2011; Guydish } \\
\text { et al., 2011; Messina et al., 2006; Miller et al., 2016; Nyamathi et al., 2018; Schram \& Morash, 2002; Scott et al., } \\
\text { 2017) }\end{array}$ \\
\hline Mixed-gendered & $2(17 \%)$ & (J. E. Johnson et al., 2011; Needels et al., 2005) \\
\hline \multicolumn{3}{|l|}{ Participants } \\
\hline Total participants & 4865 & \\
\hline Total women & 3799 & \\
\hline $\begin{array}{l}\text { Intervention participants } \\
\text { (women) }\end{array}$ & 2174 & \\
\hline $\begin{array}{l}\text { Control participants } \\
\text { (women) }\end{array}$ & 1580 & \\
\hline \multicolumn{3}{|l|}{ Participants age } \\
\hline Mean range & $30.1-39.1$ & $\begin{array}{l}\text { (Chan et al., 2005; Covington et al., 2008; Grella \& Rodriguez, 2011; Guydish et al., 2011; J. E. Johnson et al., 2011; } \\
\text { Messina et al., 2006; Miller et al., 2016; Needels et al., 2005; Nyamathi et al., 2018; Schram \& Morash, 2002; } \\
\text { Scott et al., 2017) }\end{array}$ \\
\hline Median & $31-40$ & (Schram \& Morash, 2002) \\
\hline
\end{tabular}

Identified substance use disorder

$100 \% \quad 9(75 \%)$

$\leq 100 \%$ (range $74-96 \%) \quad 3(25 \%)$

Incarceration history

Have been in prison

before this reception (\% range)

Previous contact with the criminal justice system (mean range)

Parenting characteristics

Mother

(\% range)

Average number per women

Median number per women

Intervention delivery
(Covington et al., 2008; Farrell-Macdonald et al., 2014; Grella \& Rodriguez, 2011; Guydish et al., 2011; J. E. Johnson et al., 2011; Messina et al., 2006; Miller et al., 2016; Nyamathi et al., 2018; Scott et al., 2017)

(Chan et al., 2005; Needels et al., 2005; Schram \& Morash, 2002)

55\%-92\% (Chan et al., 2005; Covington et al., 2008; Farrell-Macdonald et al., 2014; Grella \& Rodriguez, 2011; Guydish et al., 2011; Nyamathi et al., 2018; Schram \& Morash, 2002; Scott et al., 2017)

6.3-10.4 (J. E. Johnson et al., 2011; Messina et al., 2006; Miller et al., 2016; Needels et al., 2005)

$63-82 \%$

(Chan et al., 2005; Covington et al., 2008; Grella \& Rodriguez, 2011; Guydish et al., 2011; J. E. Johnson et al., 2011; Schram \& Morash, 2002; Scott et al., 2017)

1 (8\%) (Messina et al., 2006)

2 (17\%) (Chan et al., 2005) 
Table 1 Included study characteristics (Continued)

\begin{tabular}{|c|c|c|}
\hline Characteristics & N (\%) & Reference \\
\hline Post-release & $6(50 \%)$ & $\begin{array}{l}\text { (Chan et al., 2005; Covington et al., 2008; Guydish et al., 2011; J. E. Johnson et al., 2011; } \\
\text { Nyamathi et al., 2018; Scott et al., 2017) }\end{array}$ \\
\hline Transitional & $6(50 \%)$ & $\begin{array}{l}\text { (Farrell-Macdonald et al., 2014; Grella \& Rodriguez, 2011; Messina et al., 2006; Miller et al., 2016; } \\
\text { Needels et al., 2005; Schram \& Morash, 2002) }\end{array}$ \\
\hline \multicolumn{3}{|l|}{ Intervention } \\
\hline $\begin{array}{l}\text { Probation Case } \\
\text { Management }\end{array}$ & $2(17 \%)$ & (Chan et al., 2005; Guydish et al., 2011) \\
\hline $\begin{array}{l}\text { Dialectical Behavioural } \\
\text { Therapy-Corrections } \\
\text { Modified }\end{array}$ & $1(8 \%)$ & (Nyamathi et al., 2018) \\
\hline $\begin{array}{l}\text { Recovery Management } \\
\text { Check-ups }\end{array}$ & $1(8 \%)$ & (Scott et al., 2017) \\
\hline $\begin{array}{l}\text { Delaware County } \\
\text { Transition }\end{array}$ & $1(8 \%)$ & (Miller et al., 2016) \\
\hline $\begin{array}{l}\text { Methadone } \\
\text { maintenance treatment }\end{array}$ & $1(8 \%)$ & (Farrell-Macdonald et al., 2014) \\
\hline $\begin{array}{l}\text { Female Offender } \\
\text { Treatment and } \\
\text { Employment Program }\end{array}$ & $1(8 \%)$ & (Grella \& Rodriguez, 2011) \\
\hline $\begin{array}{l}\text { Collaborative Behavioral } \\
\text { Management }\end{array}$ & $1(8 \%)$ & (J. E. Johnson et al., 2011) \\
\hline $\begin{array}{l}\text { Women's Integrated } \\
\text { Treatment model }\end{array}$ & $1(8 \%)$ & (Covington et al., 2008) \\
\hline $\begin{array}{l}\text { Prison-based substance } \\
\text { abuse program and } \\
\text { community-based after- } \\
\text { care }\end{array}$ & $1(8 \%)$ & (Messina et al., 2006) \\
\hline $\begin{array}{l}\text { HealthLink jail and } \\
\text { community services }\end{array}$ & $1(8 \%)$ & (Needels et al., 2005) \\
\hline Life Skills program & $1(8 \%)$ & (Schram \& Morash, 2002) \\
\hline \multicolumn{3}{|l|}{ Comparator } \\
\hline $\begin{array}{l}\text { Standard parole/ } \\
\text { probation }\end{array}$ & $4(33 \%)$ & (Chan et al., 2005; Guydish et al., 2011; J. E. Johnson et al., 2011; Scott et al., 2017) \\
\hline $\begin{array}{l}\text { Pre-release treatment } \\
\text { group }\end{array}$ & $3(25 \%)$ & (Farrell-Macdonald et al., 2014; Messina et al., 2006; Needels et al., 2005) \\
\hline $\begin{array}{l}\text { No treatment control } \\
\text { group }\end{array}$ & $2(17 \%)$ & (Farrell-Macdonald et al., 2014; Messina et al., 2006) \\
\hline Control group not clear & $2(17 \%)$ & (Miller et al., 2016; Schram \& Morash, 2002) \\
\hline $\begin{array}{l}\text { Health Promotion } \\
\text { program }\end{array}$ & $1(8 \%)$ & (Nyamathi et al., 2018) \\
\hline $\begin{array}{l}\text { Program non- } \\
\text { completers }\end{array}$ & $1(8 \%)$ & (Grella \& Rodriguez, 2011) \\
\hline Pre/post test scores & $1(8 \%)$ & (Covington et al., 2008) \\
\hline \multicolumn{3}{|l|}{ Setting } \\
\hline Pre-release & $6(50 \%)$ & $\begin{array}{l}\text { (Farrell-Macdonald et al., 2014; Grella \& Rodriguez, 2011; Messina et al., 2006; Miller et al., 2016; } \\
\text { Needels et al., 2005; Schram \& Morash, 2002) }\end{array}$ \\
\hline Jail/prison & $4(33 \%)$ & (Farrell-Macdonald et al., 2014; Grella \& Rodriguez, 2011; Miller et al., 2016; Needels et al., 2005) \\
\hline Prison camp & $1(8 \%)$ & (Schram \& Morash, 2002) \\
\hline $\begin{array}{l}\text { Therapeutic } \\
\text { Community (in-prison } \\
\text { but separate to } \\
\text { general prison } \\
\text { population) }\end{array}$ & $1(8 \%)$ & (Messina et al., 2006) \\
\hline
\end{tabular}


Table 1 Included study characteristics (Continued)

\begin{tabular}{|c|c|c|}
\hline Characteristics & N (\%) & Reference \\
\hline Post-release & $12(100 \%)$ & $\begin{array}{l}\text { (Chan et al., 2005; Covington et al., 2008; Farrell-Macdonald et al., 2014; Grella \& Rodriguez, 2011; } \\
\text { Guydish et al., 2011; J. E. Johnson et al., 2011; Messina et al., 2006; Miller et al., 2016; Needels et al., 2005; } \\
\text { Nyamathi et al., 2018; Schram \& Morash, 2002; Scott et al., 2017) }\end{array}$ \\
\hline $\begin{array}{l}\text { Community based } \\
\text { (outpatient) }\end{array}$ & $10(83 \%)$ & $\begin{array}{l}\text { (Chan et al., 2005; Farrell-Macdonald et al., 2014; Grella \& Rodriguez, 2011; Guydish et al., 2011; } \\
\text { J. E. Johnson et al., 2011; Miller et al., 2016; Needels et al., 2005; Nyamathi et al., 2018; } \\
\text { Schram \& Morash, 2002; Scott et al., 2017) }\end{array}$ \\
\hline $\begin{array}{l}\text { Residential treatment } \\
\text { facility (inpatient) }\end{array}$ & $1(8 \%)$ & (Covington et al., 2008) \\
\hline $\begin{array}{l}\text { Post-release setting } \\
\text { not clear }\end{array}$ & $1(8 \%)$ & (Messina et al., 2006) \\
\hline \multicolumn{3}{|l|}{ Intervention length } \\
\hline \multicolumn{3}{|l|}{ Pre-release } \\
\hline $6-12$ months & $2(17 \%)$ & (Needels et al., 2005; Schram \& Morash, 2002) \\
\hline 13-24 months & $1(8 \%)$ & (Messina et al., 2006) \\
\hline $\begin{array}{l}\text { Pre-release length not } \\
\text { reported }\end{array}$ & $3(25 \%)$ & (Farrell-Macdonald et al., 2014; Grella \& Rodriguez, 2011; Miller et al., 2016) \\
\hline \multicolumn{3}{|l|}{ Post-release } \\
\hline$<3$ months & $1(8 \%)$ & (Schram \& Morash, 2002) \\
\hline $3-6$ months & $2(17 \%)$ & (J. E. Johnson et al., 2011; Messina et al., 2006) \\
\hline 7-12 months & $5(42 \%)$ & (Chan et al., 2005; Covington et al., 2008; Guydish et al., 2011; Needels et al., 2005; Nyamathi et al., 2018) \\
\hline 13-24 months & $1(8 \%)$ & (Grella \& Rodriguez, 2011) \\
\hline$>24$ months & $1(8 \%)$ & (Scott et al., 2017) \\
\hline $\begin{array}{l}\text { Pre-release length not } \\
\text { reported }\end{array}$ & $2(17 \%)$ & (Farrell-Macdonald et al., 2014; Miller et al., 2016) \\
\hline \multicolumn{3}{|l|}{ Intervention attributes } \\
\hline $\begin{array}{l}\text { Community case } \\
\text { management }\end{array}$ & $8(67 \%)$ & $\begin{array}{l}\text { (Chan et al., 2005; Grella \& Rodriguez, 2011; Guydish et al., 2011; Miller et al., 2016; Needels et al., 2005; } \\
\text { Nyamathi et al., 2018; Schram \& Morash, 2002; Scott et al., 2017) }\end{array}$ \\
\hline Gender-responsive & $7(58 \%)$ & $\begin{array}{l}\text { (Chan et al., 2005; Covington et al., 2008; Grella \& Rodriguez, 2011; Guydish et al., 2011; Nyamathi et al., 2018; } \\
\text { Schram \& Morash, 2002; Scott et al., 2017) }\end{array}$ \\
\hline Referrals to services & $7(58 \%)$ & $\begin{array}{l}\text { (Chan et al., 2005; Grella \& Rodriguez, 2011; Guydish et al., 2011; Miller et al., 2016; Needels et al., 2005; } \\
\text { Nyamathi et al., 2018; Scott et al., 2017) }\end{array}$ \\
\hline $\begin{array}{l}\text { Cognitive behavioural } \\
\text { treatment }\end{array}$ & $7(58 \%)$ & $\begin{array}{l}\text { (Covington et al., 2008; J. E. Johnson et al., 2011; Messina et al., 2006; Miller et al., 2016; Needels et al., 2005; } \\
\text { Nyamathi et al., 2018; Schram \& Morash, 2002) }\end{array}$ \\
\hline $\begin{array}{l}\text { Imbedded substance- } \\
\text { use treatment }\end{array}$ & $5(42 \%)$ & $\begin{array}{l}\text { (Covington et al., 2008; Grella \& Rodriguez, 2011; J. E. Johnson et al., 2011; Messina et al., 2006; } \\
\text { Nyamathi et al., 2018) }\end{array}$ \\
\hline $\begin{array}{l}\text { Imbedded } \mathrm{MH} \text { and/or } \\
\text { trauma services }\end{array}$ & $3(25 \%)$ & (Covington et al., 2008; Needels et al., 2005; Scott et al., 2017) \\
\hline Vocational services & $4(33 \%)$ & (Grella \& Rodriguez, 2011; Guydish et al., 2011; J. E. Johnson et al., 2011; Schram \& Morash, 2002) \\
\hline $\begin{array}{l}\text { Drug substitution } \\
\text { therapy }\end{array}$ & $1(8 \%)$ & (Farrell-Macdonald et al., 2014) \\
\hline Housing support & $1(8 \%)$ & (Schram \& Morash, 2002) \\
\hline \multicolumn{3}{|l|}{ Recidivism term used } \\
\hline Recidivism & $8(67 \%)$ & $\begin{array}{l}\text { (Farrell-Macdonald et al., 2014; Grella \& Rodriguez, 2011; Guydish et al., 2011; J. E. Johnson et al., 2011; } \\
\text { Miller et al., 2016; Nyamathi et al., 2018; Schram \& Morash, 2002; Scott et al., 2017) }\end{array}$ \\
\hline Criminal activity & $1(8 \%)$ & (Covington et al., 2008) \\
\hline Return to custody & $1(8 \%)$ & (Messina et al., 2006) \\
\hline Incarcerated & $1(8 \%)$ & (Chan et al., 2005) \\
\hline $\begin{array}{l}\text { Criminal justice system } \\
\text { involvement }\end{array}$ & $1(8 \%)$ & (Needels et al., 2005) \\
\hline
\end{tabular}


Table 1 Included study characteristics (Continued)

\begin{tabular}{|c|c|c|}
\hline Characteristics & N (\%) & Reference \\
\hline \multicolumn{3}{|l|}{ Recidivism measure } \\
\hline Return to custody & $8(67 \%)$ & $\begin{array}{l}\text { (Chan et al., 2005; Farrell-Macdonald et al., 2014; Grella \& Rodriguez, 2011; J. E. Johnson et al., 2011; } \\
\text { Messina et al., 2006; Nyamathi et al., 2018; Schram \& Morash, 2002; Scott et al., 2017) }\end{array}$ \\
\hline Re-arrest & $4(33 \%)$ & (Guydish et al., 2011; J. E. Johnson et al., 2011; Needels et al., 2005; Scott et al., 2017) \\
\hline Conviction-free & $1(8 \%)$ & (Covington et al., 2008) \\
\hline Reoffended & $1(8 \%)$ & (Miller et al., 2016) \\
\hline \multicolumn{3}{|c|}{ Reoffending post-release as a result of: } \\
\hline $\begin{array}{l}\text { Probation/parole } \\
\text { violation }\end{array}$ & $3(25 \%)$ & (Grella \& Rodriguez, 2011; Miller et al., 2016; Needels et al., 2005) \\
\hline $\begin{array}{l}\text { Charge with a new } \\
\text { crime }\end{array}$ & $3(25 \%)$ & (Grella \& Rodriguez, 2011; Miller et al., 2016; Scott et al., 2017) \\
\hline $\begin{array}{l}\text { Type of crime (drug, } \\
\text { property, violent } \\
\text { crime, prostitution) }\end{array}$ & $1(8 \%)$ & (Scott et al., 2017) \\
\hline Date of first arrest & $1(8 \%)$ & (Guydish et al., 2011) \\
\hline \multicolumn{3}{|c|}{ Follow-up time point (post-treatment) } \\
\hline 0 months & $5(42 \%)$ & (Chan et al., 2005; Guydish et al., 2011; Messina et al., 2006; Schram \& Morash, 2002; Scott et al., 2017) \\
\hline 3 months & $1(8 \%)$ & (Needels et al., 2005) \\
\hline 6 months & $3(25 \%)$ & (Covington et al., 2008; J. E. Johnson et al., 2011; Nyamathi et al., 2018) \\
\hline 12 months & $1(8 \%)$ & (Grella \& Rodriguez, 2011) \\
\hline Not reported & $2(17 \%)$ & (Farrell-Macdonald et al., 2014; Miller et al., 2016) \\
\hline \multicolumn{3}{|l|}{ Outcomes } \\
\hline Recidivism & $12(100 \%)$ & $\begin{array}{l}\text { (Chan et al., 2005; Covington et al., 2008; Farrell-Macdonald et al., 2014; Grella \& } \\
\text { Rodriguez, 2011; Guydish et al., 2011; J. E. Johnson et al., 2011; Messina et al., 2006; } \\
\text { Miller et al., 2016; Needels et al., 2005; Nyamathi et al., 2018; Schram \& Morash, 2002; Scott et al., 2017) }\end{array}$ \\
\hline Substance use & $6(50 \%)$ & $\begin{array}{l}\text { (Chan et al., 2005; Covington et al., 2008; Guydish et al., 2011; J. E. Johnson et al., 2011; } \\
\text { Needels et al., 2005; Scott et al., 2017) }\end{array}$ \\
\hline Mental Health & $4(33 \%)$ & (Chan et al., 2005; Covington et al., 2008; Guydish et al., 2011; Nyamathi et al., 2018) \\
\hline Treatment utilization & $4(33 \%)$ & (Chan et al., 2005; Guydish et al., 2011; Needels et al., 2005; Scott et al., 2017) \\
\hline HIV risk behaviours & $2(17 \%)$ & (Needels et al., 2005; Nyamathi et al., 2018) \\
\hline Social support & $2(17 \%)$ & (Chan et al., 2005; Guydish et al., 2011) \\
\hline Trauma symptomology & $1(8 \%)$ & (Covington et al., 2008) \\
\hline $\begin{array}{l}\text { Willingness/plans to } \\
\text { participate in aftercare }\end{array}$ & $1(8 \%)$ & (Grella \& Rodriguez, 2011) \\
\hline $\begin{array}{l}\text { Treatment completion } \\
\text { status }\end{array}$ & $1(8 \%)$ & (Grella \& Rodriguez, 2011) \\
\hline Child custody & $1(8 \%)$ & (Chan et al., 2005) \\
\hline Coping behaviours & $1(8 \%)$ & (Nyamathi et al., 2018) \\
\hline Client satisfaction & $1(8 \%)$ & (Covington et al., 2008) \\
\hline Discriminatory beliefs & $1(8 \%)$ & (Nyamathi et al., 2018) \\
\hline Desire for help & $1(8 \%)$ & (Nyamathi et al., 2018) \\
\hline $\begin{array}{l}\text { Survival time in the } \\
\text { community }\end{array}$ & $1(8 \%)$ & (Farrell-Macdonald et al., 2014) \\
\hline Treatment readiness & $1(8 \%)$ & (Nyamathi et al., 2018) \\
\hline Time in treatment & $1(8 \%)$ & (Grella \& Rodriguez, 2011) \\
\hline $\begin{array}{l}\text { Participation in pre- } \\
\text { release treatment }\end{array}$ & $1(8 \%)$ & (Grella \& Rodriguez, 2011) \\
\hline
\end{tabular}


outcomes post-release $(n=6)$ (Chan et al., 2005; Covington et al., 2008; Guydish et al., 2011; J. E. Johnson et al., 2011; Needels et al., 2005; Scott et al., 2017); treatment utilization $(n=4)$ (Chan et al., 2005; Guydish et al., 2011; Needels et al., 2005; Scott et al., 2017) MH outcomes $(n=4)$ (Chan et al., 2005; Covington et al., 2008; Guydish et al., 2011; Nyamathi et al., 2018), trauma symptomology $(n=1)$ (Covington et al., 2008) and child custody $(n=1)$ (Chan et al., 2005). Follow-up of women post-treatment varied between studies. Five studies captured follow-up data between 3 and 12 months postintervention (Covington et al., 2008; Grella \& Rodriguez, 2011; J. E. Johnson et al., 2011; Needels et al., 2005; Nyamathi et al., 2018), whilst five studies had no further follow-up past completion of the intervention (Chan et al., 2005; Guydish et al., 2011; Messina et al., 2006; Schram \& Morash, 2002; Scott et al., 2017) and two studies had unclear follow-up timeframes (Farrell-Macdonald et al., 2014; Miller et al., 2016).

\section{Program characteristics}

The 12 included studies assessed 11 different programs, with two studies evaluating the same intervention (Chan et al., 2005; Guydish et al., 2011). All interventions were grouped as post-release ( $n=6 ; 50 \%)$ (Chan et al., 2005; Covington et al., 2008; Guydish et al., 2011; J. E. Johnson et al., 2011; Nyamathi et al., 2018; Scott et al., 2017) or transitional $(n=6 ; 50 \%)$ (Farrell-Macdonald et al., 2014; Grella \& Rodriguez, 2011; Messina et al., 2006; Miller et al., 2016; Needels et al., 2005; Schram \& Morash, 2002) programs (Table 1 , and Table A1). One study observed the effects of methadone maintenance treatment (MMT) on opioid addicted participants (Farrell-Macdonald et al., 2014), the rest of the programs were nonpharmacological $(n=11)$ (Chan et al., 2005; Covington et al., 2008; Grella \& Rodriguez, 2011; Guydish et al., 2011; J. E. Johnson et al., 2011; Messina et al., 2006; Miller et al., 2016; Needels et al., 2005; Nyamathi et al., 2018; Schram \& Morash, 2002; Scott et al., 2017). The most common intervention attributes were community case management $(n=8)$ (Chan et al., 2005; Grella \& Rodriguez, 2011; Guydish et al., 2011; Miller et al., 2016; Needels et al., 2005; Nyamathi et al., 2018; Schram \& Morash, 2002; Scott et al., 2017), gender-responsive interventions $(n=7)$ (Chan et al., 2005; Covington et al., 2008; Grella \& Rodriguez, 2011; Guydish et al., 2011; Nyamathi et al., 2018; Schram \& Morash, 2002; Scott et al., 2017), and programs which used cognitive behavioural treatments $(n=7)$ (Covington et al., 2008; J. E. Johnson et al., 2011; Messina et al., 2006; Miller et al., 2016; Needels et al., 2005; Nyamathi et al., 2018; Schram \& Morash, 2002). Seven studies (Chan et al., 2005; Grella \& Rodriguez, 2011; Guydish et al., 2011; Miller et al., 2016; Needels et al., 2005; Nyamathi et al., 2018; Scott et al., 2017) referred women to services (SUD treatment, $\mathrm{MH}$ services, primary health care etc.) and seven had imbedded treatment services (SUD treatment (Covington et al., 2008; Grella \& Rodriguez, 2011; J. E. Johnson et al., 2011; Messina et al., 2006; Nyamathi et al., 2018), $\mathrm{MH}$ /trauma services (Covington et al., 2008; Needels et al., 2005; Scott et al., 2017)). Other attributes included vocational services $(n=4)$ (Grella \& Rodriguez, 2011; Guydish et al., 2011; J. E. Johnson et al., 2011; Schram \& Morash, 2002) and one study provided housing support (Schram \& Morash, 2002). The length of the postrelease program varied across studies from 60 days (Schram \& Morash, 2002) to three years (Scott et al., 2017) post-release, with the majority $(42 \%)$ being between 7 and 12 months post-release (Chan et al., 2005; Covington et al., 2008; Guydish et al., 2011; Needels et al., 2005; Nyamathi et al., 2018). Two studies did not report intervention length (Farrell-Macdonald et al., 2014; Miller et al., 2016).

Comparison groups were diverse. Post-release programs $(n=6)$ were compared with usual care (standard probation/parole) in 67\% of studies (Chan et al., 2005; Guydish et al., 2011; J. E. Johnson et al., 2011; Scott et al., 2017), with one of those studies (Scott et al., 2017) also conducting a within group review; one study (8.3\%) compared to a another post-release program (Nyamathi et al., 2018) and one study (8.3\%) compared participants on pre-/post-test scores (Covington et al., 2008). Transitional programs $(n=6)$ were compared to pre-release treatment groups in $50 \%$ of studies (Farrell-Macdonald et al., 2014; Messina et al., 2006; Needels et al., 2005) and two of those also compared to a no-treatment group (Farrell-Macdonald et al., 2014; Messina et al., 2006); two studies (33\%) compared against a non-specific control group (Miller et al., 2016; Schram \& Morash, 2002) and one study compared participant completers to noncompleters (Grella \& Rodriguez, 2011).

\section{Quality assessment}

The overall quality of the included studies were of a fair quality, with an average score of 0.77 (range 0.53-0.84) (See Fig. 3). Individual criteria scores ranged from 17 to $100 \%$. Missing or incomplete data was the lowest scoring item (RoB2 criteria 3.1 and 3.2 , score 0.33 ). Many studies (58\%) did not document reasons for participant drop-out (Chan et al., 2005; Covington et al., 2008; Guydish et al., 2011; Miller et al., 2016; Needels et al., 2005; Nyamathi et al., 2018; Schram \& Morash, 2002), while a minority of control groups were not clearly described (17\%) (Miller et al., 2016; Schram \& Morash, 2002), intervention length and intensity not reported (25\%) (Farrell-Macdonald et al., 2014; Grella \& Rodriguez, 2011; Miller et al., 2016) and timeframes were unclear on 


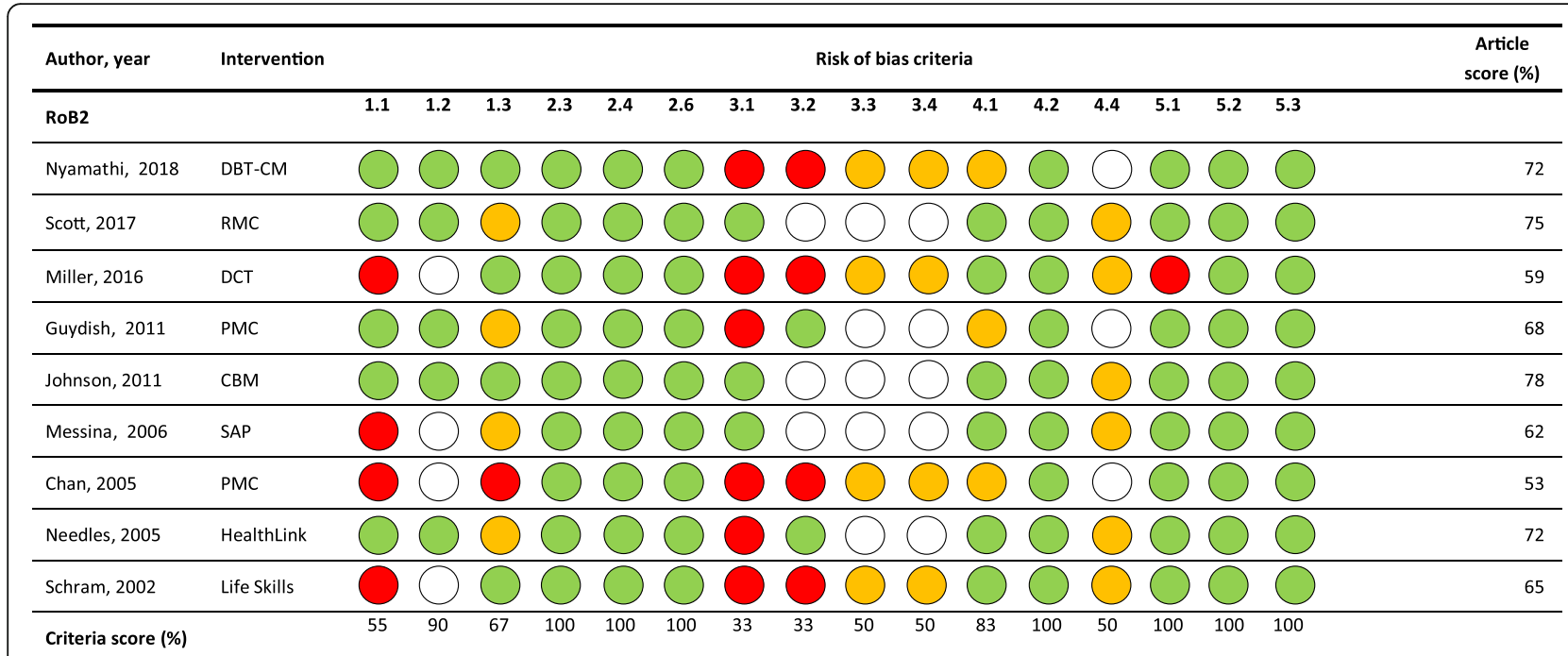

Criteria score (\%)

\begin{tabular}{|c|c|c|c|c|c|c|c|c|c|c|c|c|c|c|c|c|c|c|c|c|c|}
\hline ROBINS-I & & 1.1 & 1.2 & 1.4 & 1.5 & 1.6 & 1.7 & 1.8 & 2.1 & 2.4 & 3.1 & 3.2 & 3.3 & 4.1 & 5.1 & 5.2 & 5.3 & 6.1 & 6.3 & 6.4 & \\
\hline Farrell-M, 2014 & МMT & & & & & & & & & & & & & & & & & & & & 70 \\
\hline Grella, 2011 & FOTEP & & & & & & & & & & & & & & & & & & & & 84 \\
\hline Covington, 2008 & WIT & & & & & & & & & & & & & & & & & & & & 76 \\
\hline Criteria score (\%) & & 83 & 100 & 67 & 100 & 17 & 50 & 33 & 100 & 100 & 100 & 100 & 100 & 100 & 67 & 100 & 100 & 100 & 100 & 50 & \\
\hline
\end{tabular}

Fig. 3 Quality assessment heat map. Note: CBM - Collaborative Behavioral Management; DBT-CM - Dialectical Behavioural Therapy-Corrections Modified; DCT - the Delaware County Transition; FOTEP - the Female Offender Treatment and Employment Program; MMT - methadone maintenance treatment; PCM - Probation Case Management; RMC - Recovery Management Check-ups; RoB2 - revised Cochrane Risk of Bias tool for randomized trials; ROBINS-I - Risk of Bias in Non-randomized Studies - of Interventions; SAP -prison-based substance abuse program and community-based after-care; WIT - the Women's Integrated Treatment model

when follow-up data was captured (17\%) (Farrell-Macdonald et al., 2014; Miller et al., 2016). Allocation bias also scored low (RoB2 1.3, 0.67; ROBINS-1, 0.17 ), mainly due to major differences seen between groups at baseline (Chan et al., 2005; Covington et al., 2008; Guydish et al., 2011; Messina et al., 2006; Schram \& Morash, 2002; Scott et al., 2017).

\section{Recidivism}

The measure recidivism varied between studies and was used to quantify different crime-related events post-release (Table 2). A return-to-custody (RTC) was the most commonly used measure for recidivism $(n=8)$ (Chan et al., 2005; Farrell-Macdonald et al., 2014; Grella \& Rodriguez, 2011; J. E. Johnson et al., 2011; Messina et al., 2006; Nyamathi et al., 2018; Schram \& Morash, 2002; Scott et al., 2017). Recidivism was also a measure of re-arrest rates $(n=4)$ (Guydish et al., 2011; J. E. Johnson et al., 2011; Needels et al., 2005; Scott et al., 2017), the rate of reoffending $(n=1)$ (Miller et al., 2016) and being conviction-free at follow-up $(\mathrm{n}=1)$ (Covington et al., 2008). Six studies used more than one measure for recidivism (Grella \& Rodriguez, 2011; Guydish et al., 2011; J. E. Johnson et al., 2011; Miller et al., 2016; Needels et al., 2005; Scott et al., 2017), whereas six used a single measure (Chan et al., 2005; Covington et al., 2008; Farrell-Macdonald et al., 2014; Messina et al., 2006; Nyamathi et al., 2018; Schram \& Morash, 2002). In total, five of six transitional studies (83\%) reported significant reductions in reoffending compared to the control arm (Farrell-Macdonald et al., 2014; Grella \& Rodriguez, 2011; Messina et al., 2006; Miller et al., 2016; Schram \& Morash, 2002). Three post-release programs saw some effects: two had within group effects (Nyamathi et al., 2018; Scott et al., 2017); and one study reported reduced recidivism but lacked follow-up data to preclude significance (Covington et al., 2008).

Table 3 visually breaks down study characteristics and the correlation between recidivism outcomes. Of which five/eight (62.5\%) incorporated community case management (Grella \& Rodriguez, 2011; Miller et al., 2016; Nyamathi et al., 2018; Schram \& Morash, 2002; Scott et al., 2017); five/seven (71.4\%) reported being genderresponsive (Covington et al., 2008; Grella \& Rodriguez, 
Table 2 Study results

\begin{tabular}{|c|c|c|c|c|c|c|c|c|}
\hline \multirow[t]{2}{*}{ Author, year } & \multirow{2}{*}{$\begin{array}{l}\text { Intervention } \\
\text { vs } \\
\text { comparator }\end{array}$} & \multirow{2}{*}{$\begin{array}{l}\text { Recidivism } \\
\text { (term } \\
\text { used) }\end{array}$} & \multirow{2}{*}{$\begin{array}{l}\text { Recidivism } \\
\text { description }\end{array}$} & \multirow{2}{*}{$\begin{array}{l}\text { Follow-up } \\
\text { time post- } \\
\text { release } \\
\text { (post- } \\
\text { treatment) }\end{array}$} & \multirow{2}{*}{$\begin{array}{l}\text { Main source of } \\
\text { outcomes data }\end{array}$} & \multirow{2}{*}{$\begin{array}{l}\text { Program } \\
\text { attributes }\end{array}$} & \multicolumn{2}{|l|}{ Results } \\
\hline & & & & & & & Recidivism & $\begin{array}{l}\text { Health } \\
\text { outcomes }\end{array}$ \\
\hline \multicolumn{9}{|c|}{ Post-release programs } \\
\hline $\begin{array}{l}\text { Nyamathi, } \\
2018\end{array}$ & $\begin{array}{l}\text { DBT-CM vs } \\
\text { HP program }\end{array}$ & Recidivism & $\begin{array}{l}\text { Recidivism was } \\
\text { defined as } \\
\text { responding "Yes" } \\
\text { to the question } \\
\text { "Have you been } \\
\text { back to jail or } \\
\text { prison within the } \\
\text { past } 6 \text { months?" }\end{array}$ & $\begin{array}{l}9-15 \\
\text { months } \\
\text { post-release } \\
\text { (6 months) }\end{array}$ & $\begin{array}{l}\text { Follow-up } \\
\text { Interviews }\end{array}$ & $\begin{array}{l}1,2,3,4 \\
5\end{array}$ & 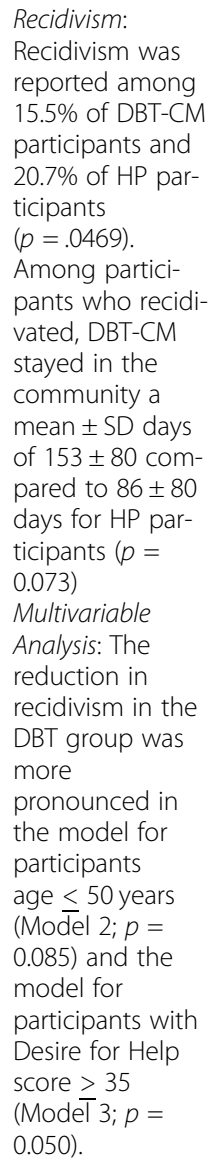 & $\begin{array}{l}\text { Substance use: } \\
\text { The majority of } \\
\text { the participants } \\
\text { reported using } \\
\text { drugs or alcohol } \\
\text { during the } 6 \\
\text { months prior to } \\
\text { the interview } \\
\text { (DBT-CM } 69.2 \% \\
\text { and } 67.7 \% \text { HP) }\end{array}$ \\
\hline Scott, 2017 & $\begin{array}{l}\text { RMC vs } \\
\text { standard } \\
\text { parole; and } \\
\text { within } \\
\text { treatment } \\
\text { group: } \\
\text { probation } \\
\text { supervision } \\
\text { vs. non- } \\
\text { probation } \\
\text { group }\end{array}$ & Recidivism & $\begin{array}{l}\text { Recidivism was } \\
\text { based on any } \\
\text { subsequent arrest } \\
\text { or incarcerations. } \\
\text { The types of } \\
\text { crimes included } \\
\text { drug crime, } \\
\text { property crime, } \\
\text { prostitution, } \\
\text { violent crime, } \\
\text { and revocation of } \\
\text { probation that } \\
\text { resulted in a } \\
\text { return to jail, } \\
\text { arrest, or new } \\
\text { charges. }\end{array}$ & $\begin{array}{l}\text { Quarterly } \\
\text { for 3-years } \\
\text { post-release } \\
\left(0^{* *}\right)\end{array}$ & $\begin{array}{l}\text { Records data } \\
\text { from Cook } \\
\text { County Jail's } \\
\text { Incarceration } \\
\text { Management } \\
\text { and Cost } \\
\text { recovery system } \\
\text { and the State of } \\
\text { Illinois' Law } \\
\text { Enforcement } \\
\text { Agencies Data } \\
\text { System, as well } \\
\text { as self-reported } \\
\text { data from the } \\
\text { GAIN }\end{array}$ & $1,2,3,6$ & $\begin{array}{l}\text { Recidivism: Total } \\
\text { percentage of } \\
\text { incarcerations } \\
\text { from baseline to } \\
36 \text { months was } \\
38 \% \text { in the RMC } \\
\text { group and } 41 \% \\
\text { in the control } \\
\text { group } \\
\text { Subject effects of } \\
\text { probation } \\
\text { supervision on } \\
\text { recidivism: } \\
\text { Women in the } \\
\text { probation group } \\
\text { were more likely } \\
\text { (i.e. worse) than } \\
\text { those in the non- } \\
\text { probation group } \\
\text { on measures of } \\
\text { new crimes (11\% } \\
\text { vs. } 9 \% ; p<0.01) ; \\
\text { new arrests or in- } \\
\text { carcerations ( } 25 \% \\
\text { vs. } 12 \% ; p<0.01) ;\end{array}$ & $\begin{array}{l}\text { Substance use: NR } \\
\text { Experimental } \\
\text { intervention } \\
\text { effects of RMC } \\
\text { (nested within } \\
\text { probation status): } \\
\text { RMCs had } \\
\text { favourable effects } \\
\text { on women in the } \\
\text { community who } \\
\text { were not on } \\
\text { probation but no } \\
\text { effect on those } \\
\text { on probation - } \\
\text { non-probation } \\
\text { women (who } \\
\text { were assigned to } \\
\text { RMC) at the be- } \\
\text { ginning of the } \\
\text { quarter were } \\
\text { more likely than } \\
\text { the control group } \\
\text { to engage in any } \\
\text { days of } \\
\text { substance-use }\end{array}$ \\
\hline
\end{tabular}


Table 2 Study results (Continued)

\begin{tabular}{llll}
\hline Author, year & $\begin{array}{l}\text { Intervention } \\
\text { vs }\end{array}$ & $\begin{array}{l}\text { Recidivism } \\
\text { (term } \\
\text { comparator }\end{array}$ & $\begin{array}{l}\text { Recidivism } \\
\text { used) }\end{array}$
\end{tabular}

\begin{tabular}{llll} 
Follow-up & Main source of & Program & Results \\
\cline { 3 - 3 } time post- & outcomes data & attributes & Recidivism \\
release & & & \\
(post- & & & \\
treatment) & & & and new crimes,
\end{tabular}

and new crimes, cerations $(33 \%$ vs. $19 \% ; p<0.01)$ Indirect effects of probation, selfhelp, and RMCs on recidivism: Treatment in the previous quarter was positively related in the subsequent quarter to the likelihood of new crimes $(\mathrm{OR}=1.76$, $p<0.01)$; new arrests or incarcerations $(\mathrm{OR}=2.19, p<$ $0.01)$; and new crimes, arrests, or incarcerations $(\mathrm{OR}=2.58, p<$ $0.01)$.

Participation in intensive selfhelp activities in the previous quarter was also related to fewer new arrests and incarcerations $(\mathrm{OR}=0.56, p<$ $0.05)$, crimes, arrests, or incarcerations $(\mathrm{OR}=0.64$ $p<0.05)$ in the next quarter. In addition, weekly alcohol and drug use was related to new crimes $(\mathrm{OR}=2.54, p<$ $0.05)$; and new crimes, arrests, or incarcerations $(\mathrm{OR}=1.28, p<$ 0.05). Finally, HIV risk behaviours were positively related to any new crimes $(\mathrm{OR}=$ $1.58, p<0.05)$ but negatively related to new arrests or incarcerations $(\mathrm{OR}=0.66, p<$ 0.05 ) and new crimes, arrests, or incarcerations $(\mathrm{OR}=0.63, p<$ $0.05)$. treatment $(8.9 \%$ vs. $4.5 \%, p<0.01)$ and in more than 10 days of treatment ( $7.5 \%$ vs. $3.9 \%, p<0.01)$. They were also less likely to engage in weekly alcohol and drug use $(47 \%$ vs. $60 \%$, $p<0.05)$, any unprotected sex (34\% vs. $46 \%$, $p<0.01)$, and any HIV risk behaviour (66\% vs. $73 \%$, $p<0.05)$. Among women on probation, none of these effects was present. Indirect effects of probation, selfhelp, and RMCs: treatment (in the previous quarter) was positively related in the subsequent quarter to weekly alcohol and drug use $(p<0.01)$. In contrast, 10 or more days of treatment $(p<$ 0.05) and participation in self-help $(p<$ $0.05)$ and intensive self-help activities $(p<0.05)$ predicted a lower likelihood of weekly alcohol and drug use. 
Table 2 Study results (Continued)

\begin{tabular}{|c|c|c|c|c|c|c|}
\hline Author, year & $\begin{array}{l}\text { Intervention } \\
\text { vs } \\
\text { comparator }\end{array}$ & $\begin{array}{l}\text { Recidivism } \\
\text { (term } \\
\text { used) }\end{array}$ & $\begin{array}{l}\text { Recidivism } \\
\text { description }\end{array}$ & $\begin{array}{l}\text { Follow-up } \\
\text { time post- } \\
\text { release } \\
\text { (post- } \\
\text { treatment) } \\
\end{array}$ & $\begin{array}{l}\text { Main source of } \\
\text { outcomes data }\end{array}$ & $\begin{array}{l}\text { Program } \\
\text { attributes }\end{array}$ \\
\hline $\begin{array}{l}\text { Guydish, } \\
2011\end{array}$ & $\begin{array}{l}\text { PCM vs } \\
\text { standard } \\
\text { probation }\end{array}$ & Recidivism & $\begin{array}{l}\text { Number of } \\
\text { arrests during the } \\
12 \text {-month follow- } \\
\text { up period and } \\
\text { date of first arrest } \\
\text { occurring in that } \\
\text { period }\end{array}$ & $\begin{array}{l}6 \text { and } 12- \\
\text { months } \\
\text { post-release } \\
\text { (0) }\end{array}$ & $\begin{array}{l}\text { San Francisco } \\
\text { integrated court } \\
\text { data } \\
\text { management } \\
\text { system }\end{array}$ & $1,2,3,7$ \\
\hline
\end{tabular}

probation (fisher's exact $=.364$ ).

Number of total

arrests: Among

those arrested at

least once, the

mean \pm SD

number of arrests

was $3.45 \pm 2.68$ in

the PCM

condition and

$3.26 \pm 2.39$ in

standard

probation

(Mann-

Whitney $=0.939$ ).

Survival analysis:

mean time to

first arrest was

$7.26 \pm 0.396$

months (for PCM

participants and

$7.08 \pm 0.369$

months for those

in standard

probation.
Health

outcomes

Substance use: NR Risk of substanceuse: PCM group has a $10 \%$ reduction in risk, relative to the standard probation, of being in the high alcohol severity category at 6 months (OR 0.90, $p=0.80$ ), however there was a $41 \%$ increase in risk at 12 months (OR $1.41, p=0.40$ ). Likewise, there was a $21 \%$ increased risk of being in the high severity drug severity group relative to standard probation (OR 1.21, $p=0.59$ ) but at 12-months PCM has a 36\%

reduction (OR $0.64, p=0.20$ ) Outcome analysis and change over time: no group effects or group by time

interactions were observed

Service Utilization: There were no significant differences between groups, at either time point or for any service measured Delivery of the PCM Intervention and Exposure Analysis: At 6 months, $53.6 \%$ of

PCM and $11.6 \%$ of standard probation participants reported having seen their PO (face-to-face meeting, one or more times)

(Fisher's exact, $p<0.0001$ ). At 12 months, the 
Table 2 Study results (Continued)

\begin{tabular}{llll}
\hline Author, year & Intervention & $\begin{array}{l}\text { Recidivism } \\
\text { (term }\end{array}$ & $\begin{array}{l}\text { Recidivism } \\
\text { description }\end{array}$ \\
& comparator & used) &
\end{tabular}

Follow-up Main source of Program Results

time post- outcomes data attributes Recidivism

release

(post-

treatment)

proportions were $43.4 \%$ and $8.5 \%$

(Fisher's exact,

$p<0.0001)$. In

the exposure

analysis,

participants who reported seeing a

PO two or more

times during 6-

12 months were

more likely to be

in the lower drug

severity category

both at 6 and 12

months ( $p=$

0.0015). The time

by case

management

interaction $(p=$

$0.74)$ shows that

this effect did not

vary by time.

Participants who

reported seeing a

PO two or more

times during the

period from 6 to

12 months were

more likely to be

in the lower

social severity

category at both

6 and 12 months

$(p=0.0366)$. The

time by case

management

interaction $(p=$

$0.63)$ shows that

this effect also

does not vary by

time.

Children $(n=100)$ :

$14.6 \%$ and $15.4 \%$

of mothers in

PCM and

standard

probation

reported living

with their

children in the

past 30 days at

the 6 month

follow-up. At the

12 month follow-

up $16.7 \%$ and

$7.5 \%$, respectively,

reporting living

with their chil-

dren in the past

30 days. At 6-

months, 23.5\%

and $20.8 \%$ partici-

pated in 
Table 2 Study results (Continued)

\begin{tabular}{|c|c|c|c|c|c|c|c|}
\hline \multirow[t]{2}{*}{ Author, year } & \multirow{2}{*}{$\begin{array}{l}\text { Intervention } \\
\text { vs } \\
\text { comparator }\end{array}$} & \multirow{2}{*}{$\begin{array}{l}\text { Recidivism } \\
\text { (term } \\
\text { used) }\end{array}$} & \multirow{2}{*}{$\begin{array}{l}\text { Recidivism } \\
\text { description }\end{array}$} & \multirow{2}{*}{$\begin{array}{l}\text { Follow-up } \\
\text { time post- } \\
\text { release } \\
\text { (post- } \\
\text { treatment) }\end{array}$} & \multirow{2}{*}{$\begin{array}{l}\text { Main source of } \\
\text { outcomes data }\end{array}$} & \multirow{2}{*}{$\begin{array}{l}\text { Program } \\
\text { attributes }\end{array}$} & Results \\
\hline & & & & & & & Recidivism \\
\hline
\end{tabular}

\begin{tabular}{|c|c|c|c|}
\hline $\begin{array}{l}\text { Johnson, } \\
2011\end{array}$ & $\begin{array}{l}\text { CBM vs } \\
\text { standard } \\
\text { parole }\end{array}$ & Recidivism & $\begin{array}{l}\text { Arrests, and } \\
\text { reincarceration } \\
\text { on a daily basis } \\
\text { during the } \\
\text { follow-up period }\end{array}$ \\
\hline
\end{tabular}

Covington, WIT model Criminal 2008 pre/post test activity

$\begin{array}{ll}\text { Percentage of } & \text { Intake, 45- } \\ \text { clients who } & \text { days, com- } \\ \text { successfully } & \text { pletion of } \\ \text { completed the } & \text { HWR and } \\ \text { program who } & \text { BT, and exit } \\ \text { reported } & \text { (6 months) } \\ \text { remaining } & \\ \begin{array}{l}\text { conviction-free at } \\ \text { follow up }\end{array} & \end{array}$
parenting classes in the past 6 months. At 12 months 20.8\% and $17 \%$ partici- pated in parent- ing classes between 6 and 12 months post- release. Even less received counsel- ling about reuni- fication, with $10.4 \%$ and $7.8 \%$ during the first 6 months post- release and $15.1 \%$ and $13.2 \%$ between 6 and 12 months post- release.

$\begin{array}{ll}3 \text { and 9- } & \text { Timeline Follow- } \\ \text { months } & \text { back calendar } \\ \text { post-release } & \text { interview } \\ \text { (6 months) } & \end{array}$
incarcerated dur-

Standardized
assessment and
program intake
form responses

Recidivism: CBM did not significantly reduce reincarceration risk - $29 \%$ of the control participants and $21 \%$ of the CBM participants were reing the 9-month follow-up.

\section{Substance use:} $17 \%$ of control participants and $11 \%$ of CBM participants used their primary drug at any time during the 9 months postrelease. When asked about alcohol only, $29 \%$ of the control participants and only $5 \%$ of the CBM participants used alcohol during this time.

\begin{tabular}{|c|c|c|}
\hline $2,4,5,6$ & $\begin{array}{l}\text { Recidivism: } 99 \% \\
\text { of the } \\
\text { participants who } \\
\text { successfully } \\
\text { completed the } \\
\text { program ( } n=40 \text { - } \\
\text { 44) reported } \\
\text { remaining } \\
\text { conviction-free } \\
\text { during the pro- } \\
\text { gram. Of those } \\
\text { who completed } \\
\text { the six-month } \\
\text { follow-up ( } n= \\
29), 97 \% \text { reported } \\
\text { not having a new } \\
\text { conviction. }\end{array}$ & $\begin{array}{l}\text { Substance use: } \\
99 \% \text { of the } \\
\text { participants who } \\
\text { successfully } \\
\text { completed the } \\
\text { program ( } \mathrm{n}=40- \\
\text { 44) reported } \\
\text { remaining drug } \\
\text { and alcohol free } \\
\text { during the } \\
\text { program. Of } \\
\text { those who } \\
\text { completed the 6- } \\
\text { month follow up } \\
\text { ( } \mathrm{n}=29 \text { ), } 72 \% \text { re- } \\
\text { ported not using } \\
\text { any alcohol or } \\
\text { other drugs since } \\
\text { exiting the } \\
\text { program. } \\
\text { Children: } 79 \% \text { had } \\
\text { dependent } \\
\text { children (age < } \\
\text { 18). Of the } 157\end{array}$ \\
\hline
\end{tabular}


Table 2 Study results (Continued)

\begin{tabular}{llll}
\hline Author, year & Intervention & $\begin{array}{l}\text { Recidivism } \\
\text { (term }\end{array}$ & $\begin{array}{l}\text { Recidivism } \\
\text { description }\end{array}$ \\
& comparator & used) &
\end{tabular}

Follow-up Main source of Program Results

time post- outcomes data attributes Recidivism Health

release

(post-

treatment) women, 52\%

planned to bring

their children

with them to the

program and

$22 \%$ reported

being pregnant at the time of

intake.

Health outcomes:

Of the 41 women

who completed

all three

assessments, the average TSC-40

score at 45 days

was $26.3 \pm 20.4$

and decreased to

a mean score of

$19.3 \pm 19.2$ after

completion of

HWR $(p<0.01)$.

The scores con-

tinued to de-

crease to a mean of $17.5 \pm 21.0$

after completion

of BT. Two sub-

scale scores

showed signifi-

cant improve-

ment between

the 45-day time

point and the

completion of

HWR: the mean

subscale score of

depression was

$6.1 \pm 4.6$ at the

45-day point and

$4.3 \pm 4.7$ after

completion of

HWR $(p<0.01)$

and the mean

subscale score of sleep distur-

bances was $6.3 \pm$ 5.4 and $4.3 \pm 4.7$

$(p<0.01)$. Anxiety and dissociation

significantly lowered between 45

days and completion of BT ( $p<$

$0.05)$. While de-

pression and

sleep distur-

bances continued

to improve with

the completion

of BT $(p<0.05)$.

Mean BDI scores

significantly de-

creased for the 
Table 2 Study results (Continued)

\begin{tabular}{|c|c|c|c|c|c|c|c|}
\hline Author, year & $\begin{array}{l}\text { Intervention } \\
\text { vs } \\
\text { comparator }\end{array}$ & $\begin{array}{l}\text { Recidivism } \\
\text { (term } \\
\text { used) }\end{array}$ & $\begin{array}{l}\text { Recidivism } \\
\text { description }\end{array}$ & $\begin{array}{l}\text { Follow-up } \\
\text { time post- } \\
\text { release } \\
\text { (post- } \\
\text { treatment) }\end{array}$ & $\begin{array}{l}\text { Main source of } \\
\text { outcomes data }\end{array}$ & $\begin{array}{l}\text { Program } \\
\text { attributes }\end{array}$ & $\begin{array}{l}\text { Results } \\
\text { Recidivism }\end{array}$ \\
\hline Chan, 2005 & $\begin{array}{l}\text { PCM vs } \\
\text { standard } \\
\text { parole }\end{array}$ & Incarcerated & $\begin{array}{l}\text { Incarcerated in } \\
\text { the } 30 \text { days } \\
\text { preceding } \\
\text { interview at } \\
\text { baseline, } 6 \\
\text { months and 12- } \\
\text { months. }\end{array}$ & $\begin{array}{l}6 \text { and } 12- \\
\text { months } \\
\text { post-release } \\
\text { (0) }\end{array}$ & $\begin{array}{l}\text { Follow-up } \\
\text { Interviews }\end{array}$ & $1,2,3$ & $\begin{array}{l}\text { Recidivism: } \\
\text { Incarceration at } 6 \\
\text { months was } 46 \% \\
\text { for PCM and } \\
55.9 \% \text { for } \\
\text { standard } \\
\text { probation and } \\
\text { was } 49.1 \% \text { and } \\
50 \% \text { at } 12 \\
\text { months, } \\
\text { respectively. }\end{array}$ \\
\hline
\end{tabular}

186 study clients (program intake) $13.8 \pm 9.3$ to 45 days $10.4 \pm 8.7$ $(p<0.05)$. In addition, scores for those clients who completed an assessment at 45 days (10.2 \pm 9.4), at completion of HWR (7.4 \pm 8.2$)$, and at the end of BT $(4.5 \pm 6.4)$ showed significant decreases at completion of each treatment component $(p<$ 0.05)

Substance use: NR Risk of substanceuse: PCM group has a 7\% increase in risk, relative to the standard probation, of being in the high alcohol severity category at 6 months (OR 1.07. $p=0.90$ ),

however there was an 8\% decrease in risk at 12 months (OR $0.92, p=0.88$ ). PCM group has a 84\% increased risk of being in the high severity drug severity group relative to standard probation (OR $1.84, p=0.31$ ) but at 12-months again a $8 \%$ reduction (OR 0.92, $p=0.88$ )

Parenting classes: $32.6 \%$ and $19.4 \%$ of PCM and standard probation groups enrolled in parenting classes in the past 6 months. Between 6 and 12 months 8.2\% PCM and $23.3 \%$ standard probation 
Table 2 Study results (Continued)

\begin{tabular}{|c|c|c|c|c|c|c|c|c|}
\hline \multirow[t]{2}{*}{ Author, year } & \multirow{2}{*}{$\begin{array}{l}\text { Intervention } \\
\text { vs } \\
\text { comparator }\end{array}$} & \multirow{2}{*}{$\begin{array}{l}\text { Recidivism } \\
\text { (term } \\
\text { used) }\end{array}$} & \multirow{2}{*}{$\begin{array}{l}\text { Recidivism } \\
\text { description }\end{array}$} & \multirow{2}{*}{$\begin{array}{l}\text { Follow-up } \\
\text { time post- } \\
\text { release } \\
\text { (post- } \\
\text { treatment) } \\
\end{array}$} & \multirow{2}{*}{$\begin{array}{l}\text { Main source of } \\
\text { outcomes data }\end{array}$} & \multirow{2}{*}{$\begin{array}{l}\text { Program } \\
\text { attributes }\end{array}$} & \multicolumn{2}{|l|}{ Results } \\
\hline & & & & & & & Recidivism & $\begin{array}{l}\text { Health } \\
\text { outcomes }\end{array}$ \\
\hline & & & & & & & & $\begin{array}{l}\text { enrolled in } \\
\text { parenting classes, } \\
\text { neither time- } \\
\text { point reached } \\
\text { significance }\end{array}$ \\
\hline \multicolumn{9}{|c|}{ Transitional programs } \\
\hline Miller, 2016 & $\begin{array}{l}\text { DCT vs } \\
\text { control group }\end{array}$ & Recidivism & $\begin{array}{l}\text { Re-offending } \\
\text { after being } \\
\text { released from } \\
\text { incarceration. } \\
\text { Three recidivism } \\
\text { variables were } \\
\text { collected: } 1 \text {. } \\
\text { probation } \\
\text { violation, } \\
\text { 2.charged with a } \\
\text { new crime, or } 3 \text {. } \\
\text { whether the } \\
\text { participant was } \\
\text { found to have } \\
\text { recidivated with } \\
\text { either a } \\
\text { probation } \\
\text { violation or a } \\
\text { new crime }\end{array}$ & $\begin{array}{l}\text { NR } \\
\text { (NR) }\end{array}$ & Survey responses & $1,3,4$ & $\begin{array}{l}\text { Recidivism: New } \\
\text { charge recidivism } \\
\text { was reported } \\
\text { among } 15.6 \% \\
\text { DCT and } 16.7 \% \\
\text { control group; } \\
\text { Probation } \\
\text { revocation } \\
\text { recidivism among } \\
28 \% \text { and } 57 \% \\
\text { ( } p<0.05) \text {, } \\
\text { respectively; and } \\
\text { any recidivism } \\
\text { among } 31 \% \text { vs. } \\
70 \% ;(p<0.01) \\
\text { Multivariate } \\
\text { logistic regression } \\
\text { models predicting } \\
\text { the odds of new } \\
\text { charge recidivism, } \\
\text { probation } \\
\text { revocation, and } \\
\text { any recidivism: } \\
\text { women in the } \\
\text { treatment group } \\
\text { were significantly } \\
\text { less likely to } \\
\text { experience any } \\
\text { recidivism relative } \\
\text { to control group } \\
(p=0.01) \text {. Being } \\
\text { married were } \\
\text { also marginally } \\
\text { less likely to } \\
\text { experience any } \\
\text { recidivism ( } p= \\
0.05 \text { ). }\end{array}$ & Substance use: NR \\
\hline $\begin{array}{l}\text { Farrell- } \\
\text { MacDonald, } \\
2014\end{array}$ & $\begin{array}{l}\text { MMT- } \\
\text { continuing vs } \\
\text { 1.terminated } \\
\text { treatment } \\
\text { and 2.no } \\
\text { treatment } \\
\text { group }\end{array}$ & Recidivism & $\begin{array}{l}\text { RTC following } \\
\text { release from } \\
\text { prison, while } \\
\text { under } \\
\text { community } \\
\text { supervision }\end{array}$ & $\begin{array}{l}27 \text { months } \\
\text { (NR) }\end{array}$ & $\begin{array}{l}\text { CSC's Offender } \\
\text { Management } \\
\text { System }\end{array}$ & 8 & $\begin{array}{l}\text { Recidivism: } 20 \% \\
\text { of the MMT-C, } \\
52 \% \text { of MMT-T, } \\
\text { and } 57 \% \text { of the } \\
\text { MMT-N group } \\
\text { had a RTC. } \\
\text { Risk of an RCT: } \\
\text { indicates that the } \\
\text { MMT-C group } \\
\text { had a } 65 \% \text { lower } \\
\text { risk of RTC than } \\
\text { the MMT-N } \\
\text { group (HR 0.35, } \\
\text { Cl 0.13-0.90). The } \\
\text { risk of RTC for } \\
\text { the MMT-T and } \\
\text { MMT-N groups } \\
\text { was not signifi- } \\
\text { cantly different } \\
\text { Type of recidivism: }\end{array}$ & Substance use: NR \\
\hline
\end{tabular}


Table 2 Study results (Continued)

\begin{tabular}{|c|c|c|c|c|c|c|c|c|}
\hline \multirow[t]{2}{*}{ Author, year } & \multirow{2}{*}{$\begin{array}{l}\text { Intervention } \\
\text { vs } \\
\text { comparator }\end{array}$} & \multirow{2}{*}{$\begin{array}{l}\text { Recidivism } \\
\text { (term } \\
\text { used) }\end{array}$} & \multirow{2}{*}{$\begin{array}{l}\text { Recidivism } \\
\text { description }\end{array}$} & \multirow{2}{*}{$\begin{array}{l}\text { Follow-up } \\
\text { time post- } \\
\text { release } \\
\text { (post- } \\
\text { treatment) }\end{array}$} & \multirow{2}{*}{$\begin{array}{l}\text { Main source of } \\
\text { outcomes data }\end{array}$} & \multirow{2}{*}{$\begin{array}{l}\text { Program } \\
\text { attributes }\end{array}$} & \multicolumn{2}{|l|}{ Results } \\
\hline & & & & & & & Recidivism & $\begin{array}{l}\text { Health } \\
\text { outcomes }\end{array}$ \\
\hline & & & & & & & $\begin{array}{l}\text { The majority of } \\
\text { RTCs in each } \\
\text { group }(60 \% \text { in } \\
\text { MMT-C, } 80 \% \text { in } \\
\text { MMT-T, } 72 \% \text { in } \\
\text { MMT-N) was re- } \\
\text { lated to technical } \\
\text { revocation. }\end{array}$ & \\
\hline Grella, 2011 & $\begin{array}{l}\text { FOTEP } \\
\text { completers vs } \\
\text { non- } \\
\text { completers }\end{array}$ & Recidivism & $\begin{array}{l}\text { Any RTP (for } \\
\text { parole violation } \\
\text { or a new charge) } \\
\text { in California over } \\
12 \text { months. }\end{array}$ & $\begin{array}{l}18-27 \\
\text { months } \\
\text { post-release } \\
\text { (12 months) }\end{array}$ & CDCR's OBIS & $\begin{array}{l}1,2,3,5 \\
7\end{array}$ & $\begin{array}{l}\text { Recidivism: } 36.8 \% \\
\text { of FOTEP } \\
\text { participants RTP } \\
\text { within } 12 \text { months } \\
\text { of FOTEP } \\
\text { discharge. Of } \\
\text { those, FOTEP } \\
\text { completers were } \\
\text { less likely to RTC } \\
\text { compared to } \\
\text { non-completers } \\
\text { at 12-months } \\
\text { post-release } \\
\text { follow-up (10.6\% } \\
\text { compared to } \\
89.4 \% ; p= \\
\text { 0.0001). } \\
\text { Type of recidivism: } \\
\text { A majority of all } \\
\text { cases were RTC } \\
\text { for a parole } \\
\text { violation (64\%), } \\
\text { 22\% were } \\
\text { returned with a } \\
\text { new term, and } \\
\text { the remainder } \\
\text { (14\%) returned } \\
\text { pending parole } \\
\text { revocation. } \\
\text { Recidivism } \\
\text { characteristics: } \\
\text { Individuals in the } \\
\text { younger age } \\
\text { groups } \\
\text { (compared to } \\
\text { older) had } \\
\text { proportionately } \\
\text { higher rates of } \\
\text { RTP. A larger } \\
\text { proportion of } \\
\text { individuals whose } \\
\text { primary } \\
\text { commitment } \\
\text { offense was } \\
\text { property-related } \\
\text { crime RTP, } \\
\text { whereas a smaller } \\
\text { proportion of } \\
\text { those with drug- } \\
\text { related offenses } \\
\text { RTP, as compared } \\
\text { with individuals } \\
\text { with violent or } \\
\text { other types of } \\
\text { offenses. } \\
\text { Survival analysis }\end{array}$ & $\begin{array}{l}\text { Substance use: NR } \\
\text { Motivation for } \\
\text { treatment: Higher } \\
\text { motivation for } \\
\text { treatment was } \\
\text { associated with } \\
\text { having a child in } \\
\text { the welfare } \\
\text { system, having } \\
\text { been in prior } \\
\text { drug treatment, } \\
\text { and using } \\
\text { "harder" drugs } \\
\text { (i.e., cocaine, } \\
\text { meth, opiates) } \\
\text { rather than } \\
\text { marijuana or } \\
\text { alcohol, as one's } \\
\text { primary } \\
\text { substance. } \\
\text { Individuals who } \\
\text { had been } \\
\text { incarcerated } \\
\text { more than once } \\
\text { were marginally } \\
\text { more likely to } \\
\text { have higher } \\
\text { motivation for } \\
\text { treatment. Lower } \\
\text { motivation for } \\
\text { treatment was } \\
\text { associated with } \\
\text { being African } \\
\text { American, } \\
\text { Hispanic, or of } \\
\text { "other" race/ } \\
\text { ethnicity, as } \\
\text { compared with } \\
\text { being White; and } \\
\text { with parole } \\
\text { region. } \\
\end{array}$ \\
\hline
\end{tabular}


Table 2 Study results (Continued)

\begin{tabular}{|c|c|c|c|c|c|c|c|c|}
\hline \multirow[t]{2}{*}{ Author, year } & \multirow{2}{*}{$\begin{array}{l}\text { Intervention } \\
\text { vs } \\
\text { comparator }\end{array}$} & \multirow{2}{*}{$\begin{array}{l}\text { Recidivism } \\
\text { (term } \\
\text { used) }\end{array}$} & \multirow{2}{*}{$\begin{array}{l}\text { Recidivism } \\
\text { description }\end{array}$} & \multirow{2}{*}{$\begin{array}{l}\text { Follow-up } \\
\text { time post- } \\
\text { release } \\
\text { (post- } \\
\text { treatment) }\end{array}$} & \multirow{2}{*}{$\begin{array}{l}\text { Main source of } \\
\text { outcomes data }\end{array}$} & \multirow{2}{*}{$\begin{array}{l}\text { Program } \\
\text { attributes }\end{array}$} & \multicolumn{2}{|l|}{ Results } \\
\hline & & & & & & & Recidivism & $\begin{array}{l}\text { Health } \\
\text { outcomes }\end{array}$ \\
\hline & & & & & & & $\begin{array}{l}\text { on RTP at } 12 \\
\text { months following } \\
\text { discharge from } \\
\text { FOTEP: There is a } \\
\text { direct linear } \\
\text { relationship } \\
\text { between time in } \\
\text { treatment and } \\
\text { risk of RTP, with } \\
\text { increasing } \\
\text { amounts of time } \\
\text { in treatment } \\
\text { associated with } \\
\text { decreasing risk of } \\
\text { RTP ( } p<0.001) \text {. } \\
\text { Other variables } \\
\text { that were } \\
\text { associated with } \\
\text { RTP were region } \\
\text { of parole, with } \\
\text { participants in } \\
\text { Region III about } \\
25 \% \text { less likely to } \\
\text { RTP than those in } \\
\text { Region I ( } p< \\
\text { 0.05). Individuals } \\
\text { who participated } \\
\text { in an in-custody } \\
\text { treatment pro- } \\
\text { gram prior to } \\
\text { their admission } \\
\text { to FOTEP were } \\
\text { about } 25 \% \text { less } \\
\text { likely to RTP } \\
\text { compared with } \\
\text { those who had } \\
\text { not ( } p<0.01 \text { ). In- } \\
\text { dividuals who } \\
\text { completed FOTEP } \\
\text { treatment were } \\
\text { about } 80 \% \text { less } \\
\text { likely to RTP } \\
\text { within } 12 \text { months } \\
\text { as compared } \\
\text { with non- } \\
\text { completers ( } p< \\
0.0001) .\end{array}$ & \\
\hline $\begin{array}{l}\text { Messina, } \\
2006\end{array}$ & $\begin{array}{l}\text { SAP + } \\
\text { community } \\
\text { after-care vs } \\
\text { 1. SAP only } \\
\text { and 2. no } \\
\text { treatment }\end{array}$ & RTC & $\begin{array}{l}\text { Percentage } \\
\text { participants who } \\
\text { RTC within six } \\
\text { months following } \\
\text { release to parole. }\end{array}$ & $\begin{array}{l}6 \text { and } 12- \\
\text { months } \\
\text { post-release } \\
\text { (0) }\end{array}$ & CDCR's OBIS & 4,5 & $\begin{array}{l}\text { Recidivism: six } \\
\text { month RCT rates } \\
\text { for SAP participants } \\
\text { were 21\%, SAP } \\
\text { and aftercare 6\% } \\
\text { and no treatment } \\
16 \% \text { ( } p<0.05) \\
\text { Multivariate } \\
\text { findings: A RTC } \\
\text { within 6-months } \\
\text { of parole was sig- } \\
\text { nificantly associ- } \\
\text { ated with age } \\
\text { and number of } \\
\text { prior incarcera- } \\
\text { tions. For each } \\
\text { additional year in }\end{array}$ & Substance use: NR \\
\hline
\end{tabular}


Table 2 Study results (Continued)

\begin{tabular}{|c|c|c|c|c|c|c|c|c|}
\hline \multirow[t]{2}{*}{ Author, year } & \multirow{2}{*}{$\begin{array}{l}\text { Intervention } \\
\text { vs } \\
\text { comparator }\end{array}$} & \multirow{2}{*}{$\begin{array}{l}\text { Recidivism } \\
\text { (term } \\
\text { used) }\end{array}$} & \multirow{2}{*}{$\begin{array}{l}\text { Recidivism } \\
\text { description }\end{array}$} & \multirow{2}{*}{$\begin{array}{l}\text { Follow-up } \\
\text { time post- } \\
\text { release } \\
\text { (post- } \\
\text { treatment) }\end{array}$} & \multirow{2}{*}{$\begin{array}{l}\text { Main source of } \\
\text { outcomes data }\end{array}$} & \multirow{2}{*}{$\begin{array}{l}\text { Program } \\
\text { attributes }\end{array}$} & \multicolumn{2}{|l|}{ Results } \\
\hline & & & & & & & Recidivism & $\begin{array}{l}\text { Health } \\
\text { outcomes }\end{array}$ \\
\hline & & & & & & & $\begin{array}{l}\text { age, the odds of } \\
\text { a } 6 \text {-month RTC } \\
\text { were decreased } \\
\text { by } 6.7 \% \text { ( } p< \\
0.01) \text {. In contrast, } \\
\text { for each add- } \\
\text { itional incarcer- } \\
\text { ation, the odds of } \\
\text { a six-month RTC } \\
\text { were increased } \\
\text { by } 21.2 \% \text { ( } p< \\
0.01) \text {. Total num- } \\
\text { ber of months in } \\
\text { aftercare treat- } \\
\text { ment approached } \\
\text { significance ( } p< \\
0.06) \text {. A RTC } \\
\text { within } 12 \text { months } \\
\text { of parole was sig- } \\
\text { nificantly associ- } \\
\text { ated with total } \\
\text { number of prior } \\
\text { incarcerations } \\
\text { and total number } \\
\text { of months in } \\
\text { community- } \\
\text { based aftercare. } \\
\text { For each additional } \\
\text { incarceration, the } \\
\text { odds of a } 12- \\
\text { month RTC were } \\
\text { increased by } 29.8 \% \\
(p<0.01) \text {. For each } \\
\text { additional month } \\
\text { in aftercare treat- } \\
\text { ment, the odds of } \\
\text { a } 12 \text {-month RTC } \\
\text { were reduced by } \\
1.5 \% \text { ( } p<0.06) \text {. } \\
\text { Prison-based treat- } \\
\text { ment/no treat- } \\
\text { ment status } \\
\text { approached signifi- } \\
\text { cance ( } p<0.08) \text {. }\end{array}$ & \\
\hline $\begin{array}{l}\text { Needels, } \\
2005\end{array}$ & $\begin{array}{l}\text { HealthLink JC } \\
\text { vs J only }\end{array}$ & $\begin{array}{l}\text { Criminal } \\
\text { Justice } \\
\text { System } \\
\text { involvement }\end{array}$ & $\begin{array}{l}\text { Rearrests or } \\
\text { parole violations }\end{array}$ & $\begin{array}{l}\text { 15-months } \\
\text { post-release } \\
\text { ( } 3 \text { months) }\end{array}$ & $\begin{array}{l}\text { Follow-up } \\
\text { Interviews }\end{array}$ & $1,3,4,6$ & $\begin{array}{l}\text { Recidivism: Events } \\
\text { resulting from } \\
\text { activity after } \\
\text { release from jail, } \\
\text { Arrested - 39\% } \\
\text { JC group and } \\
35.3 \% \text { J-only; Had } \\
\text { serious arrest } \\
\text { charge - } 1 \% \text { and } \\
4.1 \%(p<0.05) \text {, } \\
\text { respectively; Had } \\
\text { drug charge - } \\
19.5 \% \text { and } 18.4 \% \text {; } \\
\text { Convicted on at } \\
\text { least one charge } \\
-27.1 \% \text { and } \\
20.3 \% \text {; Sentenced } \\
\text { to incarceration - } \\
21.4 \% \text { and } 15.5 \% \text {; } \\
\text { Served }\end{array}$ & $\begin{array}{l}\text { Substance use: } \\
40.4 \% \text { of JC } \\
\text { group self- } \\
\text { reported drug- } \\
\text { use (any) post- } \\
\text { release, with } \\
35.5 \% \text { reporting } \\
\text { hard drugs and } \\
14.5 \% \text { reporting } \\
\text { marijuana. Simi- } \\
\text { larly, } 37.8 \% \text { of J- } \\
\text { only group self- } \\
\text { reported any } \\
\text { drug, } 31.6 \% \text { hard } \\
\text { drugs and } 18.6 \% \\
\text { marijuana. Crack } \\
\text { cocaine hair test } \\
\text { results show that } \\
26.4 \% \text { of JC } \\
\text { group and } 29.1 \%\end{array}$ \\
\hline
\end{tabular}


Table 2 Study results (Continued)

\begin{tabular}{llll}
\hline Author, year & $\begin{array}{l}\text { Intervention } \\
\text { vs }\end{array}$ & $\begin{array}{l}\text { Recidivism } \\
\text { (term } \\
\text { comparator }\end{array}$ & $\begin{array}{l}\text { Recidivism } \\
\text { used) }\end{array}$
\end{tabular}

\begin{tabular}{llll}
$\begin{array}{l}\text { Follow-up } \\
\text { time post- }\end{array}$ & $\begin{array}{l}\text { Main source of } \\
\text { outcomes data }\end{array}$ & $\begin{array}{l}\text { Program } \\
\text { attributes }\end{array}$ & Results \\
\cline { 3 - 3 } $\begin{array}{l}\text { (post- } \\
\text { treatment) }\end{array}$ & Recidivism \\
& & \\
& & incarcerated time \\
& $-35.5 \%$ and \\
& $32.6 \%$
\end{tabular}

Health

outcomes

J-only group had a negative test; $39.2 \%$ and $37.6 \%$ positive test, respectively; $1.1 \%$ and $0.8 \%$ unable to test; finally $33.3 \%$ and $32.5 \%$ unable to obtain hair sample. Post-release treatment utilization: $60 \%$ of JC participants met with their caseworkers after release; $51 \%$ of

JC participants had contact with their caseworkers at least 6 months post-release; and $36 \%$ maintained contact for most or all of the 12month eligibility period. In contrast, J-only participants were not eligible for post-release services. Caseworkers recorded an average of 6.5 h of contact during the 12-month period immediately after release, either directly with each female client or with a friend, family member, or service provider on behalf of the client.

Drug treatment: $J C$ participants (66\%) were more likely than J-only participants (56.6\%) to participate in drug treatment programs $(p<0.05)$, including the ones that provided services other than detoxification (64.4\% and $53.3 \%$, respectively) $(p<0.05)$ HIV Risk: There 
Table 2 Study results (Continued)

\begin{tabular}{|c|c|c|c|c|c|c|c|c|}
\hline \multirow[t]{2}{*}{ Author, year } & \multirow{2}{*}{$\begin{array}{l}\text { Intervention } \\
\text { vs } \\
\text { comparator }\end{array}$} & \multirow{2}{*}{$\begin{array}{l}\text { Recidivism } \\
\text { (term } \\
\text { used) }\end{array}$} & \multirow{2}{*}{$\begin{array}{l}\text { Recidivism } \\
\text { description }\end{array}$} & \multirow{2}{*}{$\begin{array}{l}\text { Follow-up } \\
\text { time post- } \\
\text { release } \\
\text { (post- } \\
\text { treatment) }\end{array}$} & \multirow{2}{*}{$\begin{array}{l}\text { Main source of } \\
\text { outcomes data }\end{array}$} & \multirow{2}{*}{$\begin{array}{l}\text { Program } \\
\text { attributes }\end{array}$} & \multicolumn{2}{|l|}{ Results } \\
\hline & & & & & & & Recidivism & $\begin{array}{l}\text { Health } \\
\text { outcomes }\end{array}$ \\
\hline & & & & & & & & $\begin{array}{l}\text { were no } \\
\text { reductions in } \\
\text { clients' self- } \\
\text { reported behav- } \\
\text { iours associated } \\
\text { with risk of HIV } \\
\text { infection }\end{array}$ \\
\hline $\begin{array}{l}\text { Schram, } \\
2002\end{array}$ & $\begin{array}{l}\text { Life Skills } \\
\text { program vs } \\
\text { comparison } \\
\text { group }\end{array}$ & Recidivism & $\begin{array}{l}\text { Woman, who } \\
\text { had been } \\
\text { released for } 60 \\
\text { days, could be } \\
\text { designated into } \\
\text { one of four } \\
\text { statuses: } 1 . \text { Not } \\
\text { returned to a } \\
\text { correctional } \\
\text { facility; } 2 \text {. } \\
\text { returned to a } \\
\text { correctional } \\
\text { facility; } 3 \text {. Still in a } \\
\text { release center or } \\
\text { on electronic } \\
\text { monitoring or; } \\
4 . \text { terminated*. }\end{array}$ & $\begin{array}{l}\text { Baseline } \\
\text { and 60-days } \\
\text { post-release } \\
\text { (0) }\end{array}$ & $\begin{array}{l}\text { Survey responses } \\
\text { and the } \\
\text { Department of } \\
\text { Corrections }\end{array}$ & $\begin{array}{l}1,2,4,7 \\
9\end{array}$ & $\begin{array}{l}\text { Recidivism: } 10 \% \\
\text { of Life Skills } \\
\text { participants } \\
\text { returned to the } \\
\text { correctional } \\
\text { system for } \\
\text { violation of } \\
\text { parole or new } \\
\text { offences in the } \\
\text { sixty day period } \\
\text { after release } \\
\text { compared to } \\
25 \% \text { for } \\
\text { comparison } \\
\text { group }(p=0.005) \text {. }\end{array}$ & $\begin{array}{l}\text { Substance use: NR } \\
\text { Group Differences } \\
\text { in Life Skills: the } \\
\text { only significant } \\
\text { differences } \\
\text { between groups } \\
\text { on post-test } \\
\text { scores were } \\
\text { Powerful Others } \\
\text { ( } p=0.058) ; \text { Cog- } \\
\text { nitive dimension } \\
\text { of the Coping } \\
\text { ( } p=0.03) \text {. } \\
\text { Differences in } \\
\text { post-tests scores } \\
\text { within treatment } \\
\text { group: Treatment } \\
\text { group } \\
\text { participants were } \\
\text { more likely to use } \\
\text { cognitive ( } p< \\
0.001), \text { social ( } p< \\
0.001), \text { spiritual } \\
\text { ( } p=0.024) \text { and } \\
\text { overall coping } \\
\text { resources ( } p= \\
0.047) \text { to handle } \\
\text { stress than they } \\
\text { had prior to } \\
\text { program } \\
\text { participation. } \\
\text { Family health and } \\
\text { nutrition: the } \\
\text { analyses } \\
\text { comparing the } \\
\text { treatment group's } \\
\text { pre/post-test } \\
\text { scores resulted the } \\
\text { participants being } \\
\text { significantly more } \\
\text { confident on the } \\
\text { post-test score that } \\
\text { they could provide } \\
\text { nutritious meals } \\
(p=0.029) \text {. }\end{array}$ \\
\hline
\end{tabular}

Note: BDI - the Beck Depression Inventory; BT - Beyond Trauma; CBM - Collaborative Behavioral Management; CDRC - California Department of Corrections and Rehabilitation; CSC - The Correctional Service of Canada; DBT-CM - Dialectical Behavioural Therapy-Corrections Modified; DCT - the Delaware County Transition; FOTEP - the Female Offender Treatment and Employment Program; GAIN - Interviews responses from the modified version of the Global Appraisal of Individual Needs; HealthLink JC -Jail and community services; HealthLink J-only - jail services only; HP - Health Promotion program; HWR - Helping Women Recover; MH - mental health; MMT-C/T/N - methadone maintenance treatment-continued/terminated/no treatment; NR - not reported; OBIS - Offender Based Information System; OR - odds ratio; PCM - Probation Case Management; PO - probation/parole officer; RMC - Recovery Management Check-ups; RTC/P - return to custody/prison; SAP + aftercare prison-based substance abuse program and community-based after-care; SAP only - prison-based substance abuse program only (pre-release); TSC-40 - the Trauma Symptom Checklist; WIT - the Women's Integrated Treatment model; 1 - Community case management; 2 -Gender responsive; 3 -Referrals to services; 4 - Cognitive behavioural treatment; 5 - Imbedded substance-use treatment; 6 - Imbedded mental health and/or trauma services; 7 - Health promotion initiatives; 8 -Drug substitution therapy; 9 - Housing support 
Table 3 Program matrix to visually depict attributes correlating to outcome change

\begin{tabular}{|c|c|c|c|c|c|c|c|c|c|c|c|}
\hline \multirow[b]{2}{*}{ Author, year } & \multirow[b]{2}{*}{ Intervention vs comparator } & \multicolumn{8}{|c|}{ Program attributes } & \multicolumn{2}{|c|}{$\begin{array}{l}\text { Outcomes } \\
\text { ( } \mathrm{S}=\text { significant and } \\
\mathrm{P}=\text { promising findings) }\end{array}$} \\
\hline & & $\begin{array}{l}\text { Post-release } \\
\text { Intervention } \\
\text { length }\end{array}$ & $\begin{array}{l}\text { Follow-up } \\
\text { post- } \\
\text { treatment }\end{array}$ & 1 & 2 & 34 & 5 & 67 & 89 & Recidivism & $\begin{array}{l}\text { Substance- } \\
\text { use }\end{array}$ \\
\hline \multicolumn{12}{|c|}{ Post-release programs } \\
\hline $\begin{array}{l}\text { Nyamathi, } \\
2018\end{array}$ & DBT-CM vs HP & $3-9 m$ & $6 \mathrm{~m}$ & $x$ & $x$ & $x \times$ & $k x$ & & & $P$ & \\
\hline Scott, 2017 & RMC vs Standard parole & $3 y$ & nil & $x$ & $x$ & $x$ & & $x$ & & $P$ & $P$ \\
\hline $\begin{array}{l}\text { Guydish, } \\
2011\end{array}$ & $\begin{array}{l}\text { PMC vs standard probation; and RMC } \\
\text { on supervision and not on supervision }\end{array}$ & $12 \mathrm{~m}$ & nil & $x$ & $x$ & $x$ & & $x$ & & & \\
\hline $\begin{array}{l}\text { Johnson, } \\
2011\end{array}$ & CBM vs standard parole & 12 weeks & $6 \mathrm{~m}$ & & & $x$ & $<x$ & $x$ & & & S \\
\hline $\begin{array}{l}\text { Covington, } \\
2008\end{array}$ & WIT pre/post test & $12 \mathrm{~m}$ & $6 \mathrm{~m}$ & & $x$ & $x$ & $k x$ & $x$ & & $P$ & $P$ \\
\hline Chan, 2005 & PMC vs standard parole & $12 \mathrm{~m}$ & nil & $x$ & $x$ & $x$ & & & & & \\
\hline \multicolumn{12}{|c|}{ Transitional programs } \\
\hline Miller, 2016 & DCT vs control group & NR & NR & $x$ & & $x \times$ & K & & & S & \\
\hline $\begin{array}{l}\text { Farrell- } \\
\text { MacDonald, } \\
2014\end{array}$ & MMT-C vs MMC-T and MMC-N & NR & $27 m^{*}$ & & & & & & $x$ & S & \\
\hline Grella, 2011 & $\begin{array}{l}\text { FOTEP completers vs FOTEP } \\
\text { non-completers }\end{array}$ & $6-15 m$ & $12 \mathrm{~m}$ & $x$ & $x$ & $x$ & $x$ & $x$ & & S & \\
\hline $\begin{array}{l}\text { Messina, } \\
2006\end{array}$ & $\begin{array}{l}\text { SAP + aftercare vs } 1 . \text { SAP only and 2. no } \\
\text { treatment }\end{array}$ & $6 \mathrm{~m}$ & $6 \mathrm{~m}$ & & & $x$ & $x \times$ & & & S & \\
\hline $\begin{array}{l}\text { Needels, } \\
2005\end{array}$ & HealthLink JC vs J-only & $12 \mathrm{~m}$ & $3 \mathrm{~m}$ & $x$ & & $x \times$ & K & $x$ & & & \\
\hline $\begin{array}{l}\text { Schram, } \\
2002\end{array}$ & Life Skills program vs comparison group & 60-days & 0 & $x$ & $x$ & $x$ & K & $x$ & $x$ & S & \\
\hline \multicolumn{12}{|c|}{$\begin{array}{l}\text { Note: CBM - Collaborative Behavioral Management; DBT-CM - Dialectical Behavioural Therapy-Corrections Modified; DCT - the Delaware County Transition; FOTEP } \\
\text { - the Female Offender Treatment and Employment Program;; HealthLink JC -Jail and community; HealthLink J-only - jail services only; HP - Health Promotion } \\
\text { program; m - months; MMT-C/T/N - methadone maintenance treatment-continuing/terminated/no treatment; nil - no follow-up past completion of the post- } \\
\text { release program; NR - not reported; P - authors concluded promising findings but results were not statistically significant; PMC - Probation Case Management; } \\
\text { RMC - Recovery Management Check-ups; S - results were statistically significant; SAP + aftercare - -prison-based substance abuse program and community-based } \\
\text { after-care; SAP only - prison-based substance abuse program only (pre-release); WIT - the Women's Integrated Treatment model; } y \text { - years; } 1 \text { - Community case } \\
\text { management; } 2 \text { - Gender responsive; } 3 \text { - Referrals to services; } 4 \text { - Cognitive behavioural treatment; } 5 \text { - Imbedded substance abuse treatment; } 6 \text { - Imbedded MH } \\
\text { and/or trauma services; } 7 \text { - Health promotion initiatives; } 8 \text { - Drug substitution therapy; } 9 \text { - Housing support } \\
\text { *Farrell-MacDonald reported that follow-up data was collect } 27 \text { months post-release but as they were unclear on intervention length we do not know how long } \\
\text { post-treatment the follow-up data was collected. For this we reported } 27 \text { months for post-treatment }\end{array}$} \\
\hline
\end{tabular}

2011; Nyamathi et al., 2018; Schram \& Morash, 2002; Scott et al., 2017); six/seven programs (85.7\%) either included or referred participants to treatment services that targeted SUDs, MH and trauma (Covington et al., 2008; Grella \& Rodriguez, 2011; Messina et al., 2006; Miller et al., 2016; Nyamathi et al., 2018; Scott et al., 2017) and five/seven (71\%) used cognitive behavioural therapies (Covington et al., 2008; Messina et al., 2006; Miller et al., 2016; Nyamathi et al., 2018; Schram \& Morash, 2002) (Tables 2 and 3). The length of the post-release component of the program (treatment in the community) varied from 60 days (Schram \& Morash, 2002) to three years (Scott et al., 2017). Two studies did not report the post-release treatment length (Farrell-Macdonald et al., 2014; Miller et al., 2016).

\section{Substance use}

Six studies (50\%) (Chan et al., 2005; Covington et al., 2008; Guydish et al., 2011; J. E. Johnson et al., 2011; Needels et al., 2005; Scott et al., 2017) examined the effect of the program on substance-use post-release, of which five $(83 \%)$ were post-release programs (Chan et al., 2005; Covington et al., 2008; Guydish et al., 2011; J. E. Johnson et al., 2011; Scott et al., 2017) and one (17\%) was transitional (Needels et al., 2005) (Tables 2 and 3 ). Three post-release programs (50\%) reported reduced substance-use at follow-up (Covington et al., 2008; J. E. Johnson et al., 2011; Scott et al., 2017). One program (J. E. Johnson et al., 2011) reported that participants in the intervention group significantly reduced substance-use post-release; one study had within group 
effects (Scott et al., 2017) and another study (Covington et al., 2008) saw reductions but lacked follow-up data to preclude significance. The attributes that supported these programs included SUD, $\mathrm{MH}$ and trauma treatment services (100\%) (Covington et al., 2008; J. E. Johnson et al., 2011; Scott et al., 2017); two programs (66.7\%) were gender-responsive (Covington et al., 2008; Scott et al., 2017), two (66.7\%) had community case management (Covington et al., 2008; Scott et al., 2017) and two (66.7\%) used cognitive behavioural therapies (Covington et al., 2008; J. E. Johnson et al., 2011). No correlation between reduced substance-use and recidivism post-release was seen.

\section{Discussion}

This is the first systematic review to examine postrelease and transitional programs offered to women with SUDs exiting prison to the community. In total we found 12 articles, which examined 11 programs, dating back to 2002, all conducted in North America. The objective of this review was to highlight the evidence about the effectiveness of post-release and transitional programs offered to women with SUDs and reveal what program attributes were common among successful programs.

The preliminary findings suggest that transitional programs had greater effects at reducing recidivism compared to post-release alone (83\% compared to $50 \%$ ). A major benefit for transitional programs is the continuity of care from prison to the community. Transitional support has been previously shown to assist participants in retaining rehabilitative health gains and reducing the risk of injury and death which is high for women with SUDs post-release (Abbot, Magin, Lujic, \& Hu, 2017; Feild, 1998; MacDonald, Williams, \& Kane, n.d.; Sullivan et al., 2019). Furthermore, as previously discussed, qualitative data also supports the use of transitional programs, as they facilitate pre-release linkage to health and social services in the community.

We were unable to make any correlations between substance-use and recidivism due to a limited pool of studies that reported substance-use as an outcome (RQ2). This is problematic considering all studies included women with SUDs and the direct correlation between substance-use and criminal offending for women is well understood (Fearn et al., 2016; H. Johnson, 2006). A major strength of this study is that it was the first to review and explore a variety of post-release and transitional programs for women with SUDs. As a result, we were able to critically examine the specific attributes of each program and make correlations between those attributes and improved post-release outcomes. Future research in this area can design or incorporate our findings into their interventions to further improve post-release outcomes for women exiting prison.

Five programs reported that allocation to the intervention group significantly reduced recidivism compared to the control group and another three concluded promising effects (RQ1). The attributes that contributed to the success of these programs were transitional, genderresponsive interventions which provided individualised support through community case management, with the use of cognitive behavioural therapies, as well as having substance-use, $\mathrm{MH}$ and trauma services available (whether it was imbedded, or women were referred to external services) (RQ3). Six studies reviewed substanceuse post-release, and of those, three reported reduced substance use among program participants (RQ1). Reductions in substance-use was associated with programs that offered gender-responsive support, used cognitive behavioural therapies, and provided substance-use treatment, $\mathrm{MH}$ and trauma services (imbedded or referred) (RQ3).

These findings reinforce the existing evidence that the design of transitional programs need to address criminogenic risk factors of women in prison (Borzycki, 2005; Borzycki \& Baldry, 2003; Carlton \& Segrave, 2016) and indicates the benefit of programs tailored to these characteristics and needs. However, we cannot determine from these studies the specifics of what was delivered to women under the banner of 'individualised support through community case management' or 'gender-responsive' interventions. In this review all studies that incorporated community case management included the role of a case manager who provided individualised links between women and external community-based services. There was no clear identification of what services women prioritised, were referred to, or managed to attend, nor the duration of attendance. Case management is the coordination of health and social services for a particular person. When employed effectively, it can bridge the services received inside prison and connect clients to appropriate community services, improving interagency information-sharing and continuity of care for individual clients (Corrective Services NSW, 2017; Feild, 1998; Warwick, Dodd, \& Neusteter, 2012). The flow on effects of improved wellbeing and rehabilitation results in increased survival-time in the community, improved health outcomes including substance-use, which ultimately improves recidivism rates for participants. These preliminary results support the use of community case management. However, further high evidence trials that clearly describe and measure the services women are referred to are needed to continue to build on the evidence pool for women exiting prison with an SUD. 
Similarly, many programs described their intervention as gender-responsive without any further description of what that involved. It should be noted that gender-responsive programming must include creating an environment through site and staff selection, and program development, content and material that reflects an understanding of the realities of the lives of women in criminal justice settings and addresses their specific challenges and strengths (Covington \& Bloom, 2006). In this review, five out of seven genderresponsive studies had an impact on recidivism. In addition, one study (Miller et al., 2016) provided community case management but did not state whether it was gender-responsive or not. It could be argued that case management is gender-responsive as it provides individualised support by linking to services based on an individual needs assessment which would target criminogenic needs, which should therefore be based on gender. This highlights two main points: 1) clearer reporting is required on what is provided when an intervention is described as genderresponsive or including case-management; and 2) Gender-responsive approaches are important and we need further research to extrapolate the aspects of gender-responsive programs that are helpful to women.

Findings from quantitative studies have shown specific attributes associated with post-release success, qualitative literature suggests there are other essential program characteristics not discussed in this review. Incarcerated women and service providers who work directly with women exiting prison have reported that stable housing, employment and family-related needs are the most critical attributes to post-release success for women (Kendall, Redshaw, Ward, Wayland, \& Sullivan, 2018; O'Brien P. \& Leem N., 2007; B. E. Salem et al., 2013). In our systematic review, no studies measured employment and housing status, or child custody in the follow-up periods. Furthermore, qualitative studies have identified the importance of continuity of care, pre-release linkage and emphasised the importance of the relationship between service providers and women participants (J. E. Johnson et al., 2013; Kendall et al., 2018; O'Brien P. \& Leem N., 2007; B. E. Salem et al., 2013). Whilst findings from our systematic review reinforce the evidence for transitional programs, they did not measure relational or acceptability aspects of program implementation. We suggest that future interventions involve key stakeholders (e.g. women with SUDs and service providers) in the program design process to get a deeper understanding of what women not only need but what attributes they want to be included in a post-release program.

\section{Limitations}

The current evidence suggests that women benefit from continuity of care from prison to the community, which incorporated gender-responsive programming and individualised case management. Generalisability is, however, limited by the fact that the majority of studies were conducted in the United States. It remains uncertain whether these programs will be effective with women in countries with a different social structure. Nevertheless, key program attributes are transferable and can inform program development.

The general scarcity of literature meant that we were unable to synthesise the true effectiveness of programs for women exiting prison with SUDs. A meta-analysis was not feasible due to the diverse range of included programs and methodological weaknesses including a lack of stringent study design and various chosen control groups, which in effect has impacted the ability to answer our research question with significance. Very few comparison groups were genuinely usual care or 'no treatment'. In most cases, the control group was receiving another program, thereby making it impossible to isolate the impact of the program under investigation.

Further, understanding the long-term impact of programs is limited due to a lack of appropriate follow-up data. Five programs did not capture data past the completion of the program. Where changes were found, there are limits to how long these changes could be assumed to last due to a lack of proper long-term followup. More research is needed on the effectiveness of post-release programs for women. They need to be of rigorous study design, with appropriate control groups and follow-up to allow evaluation of program effectiveness.

Some studies failed to report program length, frequency of intervention, and follow-up time-points. It is important to clearly describe intervention modalities so that appropriate comparisons can be made. Unexplained lost to follow-up was common among studies, with no detail on important outcomes such as program dropout, accommodation change, homelessness, rearrested/reincarceration, hospitalisation, or death. All critical to understanding the effectiveness of an intervention and recidivism. Follow-up timeframes are also an important indication of how well an intervention was able to influence participant actions post-release such as recidivism and substance-use. Many studies did not follow participants past the completion of the intervention not allowing measurement of long-term impact. A follow-up period of two years has been recommended by a number of researchers as being optimal to understand the longterm effects of a program on participants (Andersen \& Skardhamar, 2015; Office of the Inspector of Custodial Services, 2014; Yukhnenko et al., 2019). 
Recidivism is one of the most fundamental outcome measures used in criminal justice research (Duwe, 2017; King \& Elderbroom, 2014; Leverentz, Chen, Christian, \& Maruna, 2020; Urban Institute, n.d.). All studies in this review used recidivism to measure program success however, we found it was inconsistently measured and there was a lack of standardisation across studies. Another limitation relating to recidivism was that most studies used the term recidivism to express a single RTC event. This is a blunt measure, simplifying a complex series of events, failing to account for the legislative and policy context in which a RTC occurs. As a result, readers are given only a partial view of how the criminal justice system operates and the position of women within it. To make recidivism a more meaningful measure we must move beyond a single event that measures success/failure of a program. A series of events such as rearrest, reconviction and reincarceration post-release as well as desistence, time to arrest, offence type and severity (King \& Elderbroom, 2014; Urban Institute, n.d.). This suite of measures provides a timeline of events to give readers and policy makers a clearer view of the post-release experience and challenges.

Accounting for the context of health and social disadvantage experienced by women in prison, utilising health and social measures is also required. Almost all women within each study reported having a SUD prior to incarceration, however only six studies reported substanceuse post-release. Furthermore, MH, trauma, child custody, housing and employment outcomes were not analysed. This is concerning, considering the extensiveness of research illustrating these characteristics and their influence on health and recidivism post-release (Baldry, 2010; Carlton \& Segrave, 2016; Langan \& Pelissier, 2001; Sullivan et al., 2019). Future studies should include, or at least measure, these determinants in any future analysis to give a deeper understanding as to why a program was successful or not.

\section{Conclusion}

There is a paucity of literature on the effectiveness of post-release programs for women exiting prison with a SUD and the studies available contain significant methodological and conceptual limitations. There is a breadth of research that outlines the differences of characteristics of men and women within the criminal justice system, however because women make up a small proportion of the total prison population, they have received limited research attention in comparison. Recidivism rates illustrate that remaining in the community after any period in prison is difficult for women with SUDs. The rising rates of women in prison is a serious health and social policy issue in the context of what is already known about the intersecting health and social inequality experienced by women in prison and the barriers to women accessing social determinants of health resulting from disempowerment within broader social structures. The results from this review indicate that transitional, gender-responsive programs that incorporate individualised community case management and target comorbid MH and SUD can have a significant impact on post-release outcomes. Building upon these findings, development of programs for women transitioning back into the community should as a first step incorporate nuanced measures for recidivism and integrate the successful program attributes highlighted by this review.

\section{Supplementary Information}

The online version contains supplementary material available at https://doi. org/10.1186/s40352-021-00162-6.

Additional file 1. Database search strategy.

Additional file 2. Included studies.

Additional file 3. Revised Cochrane risk-of-bias tool for randomized trials (RoB 2) TEMPLATE FOR COMPLETION.

Additional file 4. The Risk Of Bias In Non-randomized Studies - of Interventions (ROBINS-I) assessment tool.

\section{Acknowledgements}

Not applicable.

\section{Authors' contributions}

$\mathrm{LE}, \mathrm{SK}, \mathrm{JB}, \mathrm{SC}$ and ES contributed to the design of the presented research. The authors developed methodology and LE and JS carried out the search strategy and quality assessment. LE extracted the data, performed the analysis, drafted the manuscript and designed the tables and figures. All authors discussed the results and provided critical review of the manuscript.

Funding

Nil

Availability of data and materials Not applicable.

\section{Declarations}

Ethics approval and consent to participate

As this study did not involve data collection from individuals but used data from published studies, ethical approval for this study was not required.

Consent for publication

Not applicable.

Competing interests

The authors declare that they have no competing interests.

\section{Author details}

${ }^{1}$ School of Public Health, Faculty of Health, University of Technology Sydney, Ultimo, NSW 2007, Australia. ${ }^{2}$ Sydney School of Education and Social Work, Faculty of Arts and Social Sciences, The University of Sydney, Sydney, NSW 2006, Australia. ${ }^{3}$ Research Operations Manager, Research Unit, Justice Health and Forensic Mental Health Network, Malabar, NSW 2036, Australia. ${ }^{4}$ Faculty of Health, University of Technology Sydney, Ultimo, NSW 2007, Australia. ${ }^{5}$ School of Public Health, Australian Centre for Public and Population Health Research, University of Technology Sydney, Ultimo, NSW 2007, Australia. ${ }^{6}$ Centre for Improving Palliative, Aged and Chronic Care through Clinical Research and Translation (IMPACCT), Faculty of Health, University of Technology Sydney, Ultimo, NSW 2007, Australia. ${ }^{7}$ College of Health, Medicine and Wellbeing, University of Newcastle, Callaghan, NSW 2308, 
Australia. ${ }^{8}$ Acting Deputy Vice Chancellor Research, University of Newcastle, Callaghan, NSW 2308, Australia. ${ }^{9}$ Custodial Health Justice Health and Forensic Mental Health Network, Malabar, NSW 2036, Australia.

Received: 29 March 2021 Accepted: 4 November 2021

Published online: 03 January 2022

\section{References}

Abbot, P., Magin, P., Lujic, S., \& Hu, W. (2017). Supporting continuity of care between prison and the community for women in prison: A medical record review. Australian Health Review, 41, 268-276. https://doi.org/10.1071/AH16007.

Alleyne, V. (2008). Locked up means locked out. Women \& Therapy, 29(3-4), 181194. https://doi.org/10.1300/J015v29n03_10.

Andersen, S. N., \& Skardhamar, T. (2015). Pick a number: Mapping recidivism measures and their consequences. Crime \& Delinquency, 63(5), 1-25. https:// doi.org/10.1177/0011128715570629.

Armstrong, K., Chartrand, V., \& Baldry, E. (2005). Submission to the antidiscrimination commissioner: For an inquiry into the Discrimination Experienced by Women Within the Criminal Justice System in New South Wales. Beyond Bars Alliance NSW. https://www.academia.edu/2166382/Submission_to_the_ NSW_Anti_Discrimination_Commissioner_for_an_Inquiry_into_the_ Discrimination_Experienced_by_Women_Within_the_Criminal_Justice_ System_in_New_South_Wales

Australian Bureau of Statistics. (2017a). Prisoners in Australia, 2017 (4517.0). Retrieved from Canberra, Australia: https://www.abs.gov.au/ausstats/abs@.nsf/ Lookup/by\%20Subject/4517.0 2017 Main\%20Features Sex 7

Australian Bureau of Statistics. (2017b). Table 1 PRISONERS, selected characteristics by most serious offence/charge (4517.0). Retrieved from: http://www.abs.gov.a u/ausstats/abs@.nsf/Lookup/by\%20Subject/4517.0 2017 Main\%20Features Key\%20findings 1

Australian Bureau of Statistics. (2018a). Corrective Services, Australia, June Quarter 2019 (4512.0). Canberra Retrieved from http://www.abs.gov.au/ausstats/abs@. $\mathrm{nsf} / \mathrm{mf} / 4512.0$

Australian Bureau of Statistics. (2018b). Corrective Services, Australia, September Quarter 2020 (4512.0). Retrieved from Canberra: http://www.abs.gov.au/aussta ts/abs@.nsf/mf/4512.0

Australian Institute of Family Studies. (2012). Addressing women's victimisation histories in custodial settings. (no. 13). Canberra: Australian Government Retrieved from https://aifs.gov.au/publications/addressing-womens-victimisa tion-histories-custodial-settings/profile-women-prison

Australian Institute of Health and Welfare (2019). The health of Australia's prisoners 2018, Cat. no. PHE 246 (). AlHW: Canberra.

Back, S. E., Payne, R. L., Wahlquist, A. H., Carter, R. E., Stroud, Z., Haynes, L., ... Ling, W. (2011). Comparative profiles of men and women with opioid dependence: Results from a national multisite effectiveness trial. The American Journal of Drug and Alcohol Abuse, 37(5), 313-323. https://doi.org/1 0.3109/00952990.2011.596982.

Baldry, E. (2010). Women in Transition: From Prison to... Current. Issues in Criminal Justice, 22(2), 253-267.

Baldry, E., McDonnell, D., Maplestone, P., \& Peeters, M. (2006). Ex-prisoners, homelessness and the state in Australia. The Australian and New Zealand Journal of Criminology, 39(1), 20-33. https://doi.org/10.1375/acri.39.1.20.

Balyakina, E., Mann, C., Ellison, M., Sivernell, R., Fulda, K. G., Sarai, S. K., \& Cardarelli, R. (2014). Risk of future offense among probationers with co-occurring substance use and mental health disorders. Community Mental Health Journal, 50(3), 288-295. https://doi.org/10.1007/s10597-013-9624-4.

Bartels, L., \& Gaffney, A. (2011). Good practice in women's prisons: A literature review. Technical and background paper series no. 41. Canberra: Australian Institute of Criminology. https://www.aic.gov.au/publications/tbp/tbp41

Begun, A. L., Early, T. J., \& Hodge, A. (2016). Mental health and substance abuse service engagement by men and women during community reentry following incarceration. Administration and Policy in Mental Health, 43(2), 207218. https://doi.org/10.1007/s10488-015-0632-2.

Begun, A. L., Rose, S. J., \& LeBel, T. P. (2011). Intervening with women in jail around alcohol and substance abuse during preparation for community reentry. Alcoholism Treatment Quarterly, 29(4), 453-478. https://doi.org/10.1 080/07347324.2011.608333.

Bello, J. K., Hearing, C., Salas, J., Weinstock, J., \& Linhorst, D. (2020). Trends in substance use by gender among participants in a jail-based substance use disorder treatment program: 1998-2016. Journal of Forensic Sciences, 65(1), 97-102. https://doi.org/10.1111/1556-4029.14153.
Borzycki, M. (2005). Interventions for Prisoners Returning to the Community Retrieved from Canberra: https:/aic.gov.au/publications/archive/ interventions-for-prisoners-returning-to-the-community

Borzycki, M., \& Baldry, E. (2003). Promoting integration: the provision of prisoner post-release services. Trends \& issues in crime and criminal justice no. 262. Canberra: Australian Institute of Criminology. https://www.aic.gov.au/publica tions/tandi/tandi262

Carlton, B., \& Segrave, M. (2016). Rethinking women's post-release reintegration and 'success'. Australian \& New Zealand Journal of Criminology, 49(2), 281299. https://doi.org/10.1177/0004865815573876.

Chan, M., Guydish, J., Prem, R., Jessup, M. A., Cervantes, A., \& Bostrom, A. (2005). Evaluation of probation case management (PCM) for drug-involved women offenders. Crime \& Delinquency, 51(4), 447-469. https://doi.org/10.1177/ 0011128704273580.

Corrective Services NSW. (2017). Policy for case Management in Correctional Centres. Retrieved from

Covington, S. S. (2001). A Woman's journey home: Challenges for female offenders and their children [Paper presentation]. The From Prisons to Home Conference: The Effect of Incarceration and Reentry on Children, Families, and Communities. https://aspe.hhs.gov/basic-report/womans-journey-homechallenges-female-offenders-and-their-children

Covington, S. S., \& Bloom, B. E. (2006). Gender-responsive treatment and Services in Correctional Settings. Women and Therapy, 29(3/4), 9-33.

Covington, S. S., Burke, C., Keaton, S., \& Norcott, C. (2008). Evaluation of a traumainformed and gender-responsive intervention for women in drug treatment. Journal of psychoactive drugs, 40(sup5), 387-398. https://doi.org/10.1080/02 791072.2008.10400666.

Dumont, D. M., Brockmann, B., Dickman, S., Alexander, N., \& Rich, J. D. (2012). Public health and the epidemic of incarceration. Annual Review of Public Health, 33(1), 325-339. https://doi.org/10.1146/annurev-publhealth-031811-124614.

Duwe, G. (2017). The use and impact of correctional programming for inmates on pre- and post-release outcomes. (NCJ 250476). Minnesota Department of Corrections: U.S. Department of Justice.

Farrell-Macdonald, S., Macswain, M. A., Cheverie, M., Tiesmaki, M., \& Fischer, B. (2014). Impact of methadone maintenance treatment on women offenders post-release recidivism. European Addiction Research, 20(4), 192-199. https:// doi.org/10.1159/000357942.

Fazel, F., Yoon, I. A., \& Hayes, A. J. (2017). Substance use disorders in prisoners: An updated systematic review and meta-regression analysis in recently incarcerated men and women. Addiction, 122(10), 1725-1739. https://doi. org/10.1111/add.13877.

Fearn, N. E., Vaughn, M. G., Nelson, E. J., Salas-Wright, C. P., DeLisi, M., \& Qian, Z. (2016). Trends and correlates of substance use disorders among probationers and parolees in the United States, 2002-2014. Drug and Alcohol Dependence, 167, 128-139. https://doi.org/10.1016/j.drugalcdep.2 016.08.003.

Feild, G. (1998). Continuity of offender treatment: Institution to the community. (NC) Number: 171833). Office of National Drug Control Policy. https://www.ojp. gov/ncjrs/virtual-library/abstracts/continuity-offender-treatment-institutioncommunity

Gobeil, R., Blanchette, K., \& Stewart, L. (2016). A Meta-analytic review of correctional interventions for women offenders: Gender-neutral versus gender-informed approaches. Criminal Justice and Behavior, 43(3), 301-322. https://doi.org/10.1177/0093854815621100.

Grella, C. E., \& Rodriguez, L. (2011). Motivation for treatment among women offenders in prison-based treatment and longitudinal outcomes among those who participate in community aftercare. Journal of psychoactive drugs, 43(sup1), 58-67. https://doi.org/10.1080/02791072.2 011.602275.

Guydish, J., Chan, M., Bostrom, A., Jessup, M. A., Davis, T. B., \& Marsh, M. (2011). A randomized trial of probation case management for drug-involved women offenders. Crime \& Delinquency, 57(2), 167-198. https://doi.org/10.1177/ 0011128708318944

Hoffmann, T. C., Glasziou, P. P., Boutron, I., Milne, R., Perera, R., Moher, D. ... Michie, S. (2014). Better reporting of interventions: Template for intervention description and replication (TIDieR) checklist and quide. BMJ, 348(mar07 3), g1687. https://doi.org/10.1136/bmj.g1687.

Hughes, K., Bellis, M. A., Hardcastle, K. A., Sethi, D., Butchart, A., Mikton, C..... Dunne, M. P. (2017). The effect of multiple adverse childhood experiences on health: A systematic review and meta-analysis. The Lancet Public Health, 2(8), e356-e366. https://doi.org/10.1016/S2468-2667(17)30118-4. 
Johnson, H. (2006). Trends \& issues in crime and criminal justice: Factors associated with drug and alcohol dependency among women in prison (Report no. 318). Australian Institute of Criminology. https://aic.gov.au/publications/tandi/tandi318.

Johnson, J. E., Friedmann, P. D., Green, T. C., Harrington, M., \& Taxman, F. S. (2011) Gender and treatment response in substance use treatment-mandated parolees. Journal of Substance Abuse Treatment, 40(3), 313-321. https://doi. org/10.1016/j.jsat.2010.11.013.

Johnson, J. E., Schonbrun, Y. C., Nargiso, J. E., Kuo, C. C., Shefner, R. T., Williams, C. A., \& Zlotnick, C. (2013). "I know if I drink I won't feel anything": Substance use relapse among depressed women leaving prison. International Journal of Prisoner Health, 9(4), 169-186. https://doi.org/10.1108/ijph-02-2013-0009.

Johnson, J. E., \& Zlotnick, C. (2008). A pilot study of group interpersonal psychotherapy for depression in substance-abusing female prisoners. Journal of Substance Abuse Treatment, 34(4), 371-377. https://doi.org/10.1016/j.jsat.2 007.05.010.

Karlsson, M. E., \& Zielinski, M. J. (2018). Sexual victimization and mental illness prevalence rates among incarcerated women: A literature review. Trauma, Violence, \& Abuse, 21(2), 326-349. https://doi.org/10.1177/1524838018767933.

Kendall, S., Redshaw, S., Ward, S., Wayland, S., \& Sullivan, E. (2018). Systematic review of qualitative evaluations of reentry programs addressing problematic drug use and mental health disorders amongst people transitioning from prison to communities. Health \& Justice, 6(1), 4. https://doi.org/10.1186/s403 52-018-0063-8.

Kilroy, D. (2016). Women in prison in Australia. Paper presented at the current issues in sentencing conference. Canberra: Australian National University.

King, R., \& Elderbroom, B. (2014). Improving recidivism as a performance measure. DC: Retrieved from Washington.

Kinner, S. A., \& Young, J. T. (2018). Understanding and improving the health of people who experience incarceration: An overview and synthesis. Epidemiologic Reviews, 40(1), 4-11. https://doi.org/10.1093/epirev/mxx018.

Langan, N. P., \& Pelissier, B. M. (2001). Gender differences among prisoners in drug treatment. Journal of Substance Abuse, 13(3), 291-301. https://doi.org/1 0.1016/S0899-3289(01)00083-9.

Lawlor, E., Nicholls, J., \& Sanfilippo, L. (2008). Unlocking value: How we all benefit from investing in alternatives to prison for women offenders. London: New Economics Foundation Retrieved from https://neweconomics.org/uploads/ files/6533e0a823956802b3_10m6buwft.pdf.

Leverentz, A., Chen, E. Y., Christian, J., \& Maruna, S. (2020). Beyond recidivism: New approaches to research on prisoner reentry and reintegration. https://doi.org/1 0.18574/nyu/9781479862726.001.0001.

MacDonald, M., Williams, J., \& Kane, D. Throughcare for prisoners with problematic drug use: a European perspective. EuroVista, 2(3), 144-153.

Messina, N., Burdon, W., \& Prendergast, M. (2006). Prison-based treatment for drug-dependent women offenders: Treatment versus no treatment. Journal of psychoactive drugs, 38(sup3), 333-343. https://doi.org/10.1080/02791072.2 006.10400597.

Messina, N., \& Grella, C. E. (2006). Childhood trauma and women's health outcomes in a California prison population. American Journal of Public Health, 96(10), 1842-1848. https://doi.org/10.2105/AJPH.2005.082016.

Miller, H. V., Miller, J. M., \& Barnes, J. C. (2016). Reentry programming for opioid and opiate involved female offenders: Findings from a mixed methods evaluation. Journal of Criminal Justice, 46, 129-136. https://doi.org/10.1016/j. jcrimjus.2016.04.001.

Moher, D., Liberati, A., Tetzlaff, J., Altman, D. G., \& the PRISMA Group (2009). Preferred reporting items for systematic reviews and meta-analyses: The PRISMA statement. British Medical Journal, 339, b2535. https://doi.org/10.113 6/bmj.b2535.

Moore, K. E., Hacker, R. L., Oberleitner, L., \& McKee, S. A. (2020). Reentry interventions that address substance use: A systematic review. Psychological Services, 17(1), 93-101. https://doi.org/10.1037/ser0000293.

Needels, K., James-Burdumy, S., \& Burghardt, J. (2005). Community case management for former jail inmates: Its impacts on rearrest, drug use, and HIV risk. Journal of Urban Health, 82(3), 420-433. https://doi.org/10.1093/jurba $\mathrm{n} / \mathrm{jti092.}$

NIDA. (2021). Sex and Gender Differences in Substance Use. Retrieved from https://www.drugabuse.gov/publications/research-reports/substance-use-inwomen/sex-gender-differences-in-substance-use

Nyamathi, A., Shin, S. S., Smeltzer, J., Salem, B., Yadav, K., Krogh, D., \& Ekstrand, M. (2018). Effectiveness of dialectical behavioral therapy on reduction of recidivism among recently incarcerated homeless women: A pilot study.
International Journal of Offender Therapy and Comparative Criminology, 62(15), 4796-4813. https://doi.org/10.1177/0306624X18785516.

O'Brien, P., \& Leem, N. (2007). Moving from needs to self-efficacy: A holistic system for women in transition from prison. Women \& Therapy, 29(3), 261284. https://doi.org/10.1300/J015v29n03_14.

Office of the Inspector of Custodial Services (2014). Recidivism rates and the impact of treatment programs. (ISSN 1445-3134). Perth, WA: Government of Western Australia.

Pelissier, B. M., Motivans, M., \& Rounds-Bryant, J. L. (2005). Substance abuse treatment outcomes: A multi-site study of male and female prison programs. Journal of Offender Rehabilitation, 41(2), 57-80. https://doi.org/10.1300/ J076v41n02_04.

Ray, B., Grommon, E., Buchanan, V., Brown, B., \& Watson, D. P. (2017). Access to recovery and recidivism among former prison inmates. International Journal of Offender Therapy and Comparative Criminology, 61(8), 874-893. https://doi. org/10.1177/0306624X15606688.

Rushforth, C., \& Willis, K. (2003). The female criminal: An overview of women's drug use and offending behaviour. (no. 264.). Retrieved from Canberra: Australian Institute of Criminology. : https://www.aic.gov.au/publications/tandi/tandi264

Salem, B. E., Nyamathi, A., Idemundia, F., Slaughter, R., \& Ames, M. (2013). At a crossroads: Reentry challenges and healthcare needs among homeless female ex-offenders. Journal of Forensic Nursing, 9(1), 14-22. https://doi.org/1 0.1097/jfn.0b013e31827a1e9d.

Schonbrun, Y. C., Johnson, J. E., Anderson, B. J., Caviness, C., \& Stein, M. D. (2017). Hazardously drinking jailed women: Post-release perceived needs and risk of Reincarceration. International Journal of Offender Therapy and Comparative Criminology, 61(16), 1819-1832. https://doi.org/10.1177/03 $06624 \times 16634702$.

Schram, P. J., \& Morash, M. (2002). Evaluation of a life skills program for women inmates in Michigan. Journal of Offender Rehabilitation, 34(4), 47-70. https:// doi.org/10.1300/J076v34n04_03.

Scott, C. K., Dennis, M. L., \& Lurigio, A. J. (2017). The effects of specialized probation and recovery management checkups (RMCs) on treatment participation, substance use, HIV risk behaviors, and recidivism among female offenders: Main findings of a 3-year experiment using subject by intervention interaction analysis. Journal of Experimental Criminology, 13(1), 53-77. https:// doi.org/10.1007/s11292-016-9281-z.

Segrave, M., \& Carlton, B. (2011). Counting the costs of imprisonment: Researching women's post-release deaths in Victoria. Australian \& New Zealand Journal of Criminology, 44(1), 41-55. https://doi.org/10.1177/0004 865810392868

Stalans, S. J. (2009). Women's offending behavior: Evidence-based review of gender differences and gender responsive programs. Victims and Offenders, 4(4), 405-411. https://doi.org/10.1080/15564880903227545.

Sterne, J. A. C., Hernán, M. A., Reeves, B. C., Savović, J., Berkman, N. D., Viswanathan, M., ... JPT, H. (2016). ROBINS-I: A tool for assessing risk of bias in non-randomized studies of interventions. BMJ, 355, i4919. https://doi.org/1 0.1136/bmj.i4919.

Sterne, J. A. C., Savović, J., Page, M. J., Elbers, R. G., Blencowe, N. S., Boutron, I., ... JPT., H. (2019). RoB 2: A revised tool for assessing risk of bias in randomised trials. BMJ Open, 366, 14898. https://doi.org/10.1136/bmj.14898.

Sullivan, E., Ward, S., Zeki, R., Wayland, S., Sherwood, J., Wang, A., ... Chang, S. (2019). Recidivism, health and social functioning following release to the community of NSW prisoners with problematic drug use: Study protocol of the population-based retrospective cohort study on the evaluation of the connections program. BMJ Open, 9(7), e030546. https://doi.org/10.1136/ bmjopen-2019-030546.

Suter, J. M., Byrne, M. K., Byrne, S., Howells, K., \& Day, A. (2002). Anger in prisoners: Women are different from men. Personality and Individual Differences, 32(6), 1087-1100. https://doi.org/10.1016/S0191-8869(01)00105-2.

Tuchman, E. (2010). Women and addiction: The importance of gender issues in substance abuse research. Journal of Addictive Diseases, 29(2), 127-138. https://doi.org/10.1080/10550881003684582.

Urban Institute. (n.d). Measuring recidivism at the local level: A quick guide. Retrieved from https://www.urban.org/sites/default/files/recidivism-mea sures_final-forwebsite.pdf $+\& c d=1 \& h|=e n \& c t=c| n k \& g l=a u \& c l i e n t=$ firefoxb-e

van den Bergh, B. J., Gatherer, A., \& Møller, L. F. (2009). Women's health in prison: Urgent need for improvement in gender equity and social justice. Bulletin of the World Health Organization, 87(6), 406. https://doi.org/10.2471/blt.09.066928. 
Walmsley, R. (2017). World Female Imprisonment List: fourth edition. Retrieved from London: https://www.prisonstudies.org/news/world-female-imprisonmentlist-fourth-edition

Warwick, K., Dodd, H., \& Neusteter, R. (2012). Case management strategies for successful jail reentry. Retrieved from Washington: https://nicic.gov/case-mana gement-strategies-successful-jail-reentry

Wetton, C., \& Sprackett, J. (2007). Women in prison: A review of the conditions in member states of the Council of Europe. In Executive summany Retrieved from www.quaker.org/qcea.

Women in Prison. (2017). Key facts. A round-up and latest key statistics regarding women affected by the criminal justice system. Retrieved from https://www. womeninprison.org.uk/research/key-facts.php

World Health Organisation. (2009). Women's Health in Prison: Correcting Gender Inequity in Prison Health. Retrieved from http://www.euro.who.int/data/a ssets/pdf file/0004/76513/E92347.pdf

World Prison Brief. (2018). World Prison Brief data: United States of America. Retrieved from https://www.prisonstudies.org/country/united-states-america

Yukhnenko, D., Sridhar, S., \& Fazel, S. (2019). A systematic review of criminal recidivism rates worldwide: 3-year update. Wellcome Open Research, 4, 28 https://doi.org/10.12688/wellcomeopenres.14970.1.

\section{Publisher's Note}

Springer Nature remains neutral with regard to jurisdictional claims in published maps and institutional affiliations.

Ready to submit your research? Choose BMC and benefit from:

- fast, convenient online submission

- thorough peer review by experienced researchers in your field

- rapid publication on acceptance

- support for research data, including large and complex data types

- gold Open Access which fosters wider collaboration and increased citations

- maximum visibility for your research: over $100 \mathrm{M}$ website views per year

At BMC, research is always in progress.

Learn more biomedcentral.com/submissions 